\title{
Analysis of Implicit Type Nonlinear Dynamical Problem of Impulsive Fractional Differential Equations
}

\author{
Naveed Ahmad, ${ }^{1}$ Zeeshan Ali, ${ }^{2}$ Kamal Shah $\mathbb{D},{ }^{3}$ Akbar Zada $\mathbb{D}^{2},{ }^{2}$ and Ghaus ur Rahman ${ }^{4}$ \\ ${ }^{1}$ Institution of Business Administration, University Road, Karachi 74200, Pakistan \\ ${ }^{2}$ Department of Mathematics, University of Peshawar, Peshawar, Khyber Pakhtunkhwa, Pakistan \\ ${ }^{3}$ Department of Mathematics, University of Malakand, Dir(L), Khyber Pakhtunkhwa, Pakistan \\ ${ }^{4}$ Department of Mathematics and Statistics, University of Swat, Mingora, Khyber Pakhtunkhwa, Pakistan
}

Correspondence should be addressed to Kamal Shah; kamalshah408@gmail.com

Received 21 November 2017; Accepted 10 January 2018; Published 12 February 2018

Academic Editor: Maricel Agop

Copyright (C) 2018 Naveed Ahmad et al. This is an open access article distributed under the Creative Commons Attribution License, which permits unrestricted use, distribution, and reproduction in any medium, provided the original work is properly cited.

We study the existence, uniqueness, and various kinds of Ulam-Hyers stability of the solutions to a nonlinear implicit type dynamical problem of impulsive fractional differential equations with nonlocal boundary conditions involving Caputo derivative. We develop conditions for uniqueness and existence by using the classical fixed point theorems such as Banach fixed point theorem and Krasnoselskii's fixed point theorem. For stability, we utilized classical functional analysis. Also, an example is given to demonstrate our main theoretical results.

\section{Introduction}

Over the past few decades, differential equations of fractional order have got considerable attention from the researchers due to their significant applications in various disciplines of science and technology. Fractional derivatives introduce amazing instrument for the description of general properties of different materials and processes. This is the primary advantage of fractional derivatives in comparison with classical integer order models, in which such impacts are in fact ignored. The advantages of fractional derivatives become apparent in modeling mechanical and electrical properties of real materials, as well as in the description of properties of gases, liquids, rocks and in many other fields (see $[1,2]$ ). Since fractional order differential equations play important roles in modeling real world problems related to biology, viscoelasticity, physics, chemistry, control theory, economics, signal and image processing phenomenon, bioengineering, and so forth (for details, see [3-7]), it is investigated that fractional order differential equations model real world problems more accurately than differential equations of integer order. The area devoted to the study of existence and uniqueness of solutions to initial/boundary value problems for fractional order differential equations has been studied very well and plenty of papers are available on it in the literature. We refer the reader to few of them in [8-14] and the references therein. To model evolution process and phenomena which are experienced from sudden changes in their states, impulsive differential equations serve as a powerful mathematical tool to model them. In daily life, we observe several physical systems that suffer from impulsive behavior such as the pendulum clock action, heart function, mechanical systems subject to impacts, dynamic of system with automatic regulation, the maintenance of a species through periodic stocking or harvesting, the thrust impulse maneuver of a spacecraft, control of the satellite orbit, disturbances in cellular neural networks, fluctuations of economical systems, vibrations of percussive systems, and relaxational oscillations of the electromechanical systems. For details, see [15-23].

In some cases, nonlocal conditions are imposed instead of local conditions. It is sometimes better to impose nonlocal conditions since the measurements needed by a nonlocal condition may be more precise than the measurement given by a local condition in dynamical problems. Also, nonlocal boundary value problems have become an expeditiously 
growing area of research. The study of this type of problems is driven not only by a theoretical interest, but also by the fact that several phenomena in physics, engineering, and life sciences can be modeled in this manner, for example, problems with feedback controls such as the steady-states of a thermostat, where a controller at one of its ends adds or removes heat. For more applications, see [24] and references therein. Due to the aforesaid significant behavior, we prefer to take nonlocal boundary conditions.

The definitions of the fractional order derivative are not unique and there exist several definitions, including Grunwald-Letnikov [7], Riemann-Liouville [25], WeylRiesz [26], Erdlyi-Kober [27], and the Caputo [28] representation for fractional order derivative. In the Caputo case, the derivative of a constant is zero and we can define, properly, the initial conditions for the fractional differential equations which can be handled by using an analogy with the classical integer case. For these reasons, in this manuscript, we prefer to use the Caputo fractional derivative.

Tian and Bai [29] studied the existence and uniqueness of the following nonlinear impulsive boundary value problem:

$$
\begin{aligned}
{ }^{c} D^{\rho} y(t) & =f(t, y(t)), \\
t \in[0,1], t \neq t_{j}, j=1,2, \ldots, k, & \\
\Delta y\left(t_{j}\right) & =I_{j}\left(y\left(t_{j}\right)\right), \\
\Delta y^{\prime}\left(t_{j}\right) & =\widehat{I}_{j}\left(y\left(t_{j}\right)\right), \\
y(0) & =g(y), \\
y(1) & =h(y),
\end{aligned}
$$

where $1<\rho \leq 2, f:[0,1] \times \mathbb{R} \rightarrow \mathbb{R}$ is continuous, $I_{j}, \widehat{I}_{j}$ : $\mathbb{R} \rightarrow \mathbb{R}$ are continuous, and

$$
\begin{gathered}
\Delta\left(y\left(t_{j}\right)\right)=y\left(t_{j}^{+}\right)-y\left(t_{j}^{-}\right), \\
\Delta\left(y^{\prime}\left(t_{j}\right)\right)=y^{\prime}\left(t_{j}^{+}\right)-y^{\prime}\left(t_{j}^{-}\right)
\end{gathered}
$$

with $y\left(t_{j}^{+}\right), y^{\prime}\left(t_{j}^{+}\right), y\left(t_{j}^{-}\right), y^{\prime}\left(t_{j}^{-}\right)$are the respective left and right limits of $y\left(t_{j}\right)$ at $t=t_{j}$.

In 1940, Ulam posed the following problem about the stability of functional equations: "Under what conditions does there exist an additive mapping near an approximately additive mapping?" (see [30]). In the following year, Hyers gave an answer to the problem of Ulam for additive functions defined on Banach spaces [31]. Let $\mathscr{B}_{1}, \mathscr{B}_{2}$ be two real Banach spaces and $\epsilon>0$. Then for each mapping $f: \mathscr{B}_{1} \rightarrow \mathscr{B}_{2}$ satisfying

$$
\|f(y+z)-f(y)-f(z)\| \leq \epsilon
$$

for all $y, z \in \mathscr{B}_{1}$, there is a unique additive mapping $g$ : $\mathscr{B}_{1} \rightarrow \mathscr{B}_{2}$ with

$$
\|f(y)-g(y)\| \leq \epsilon, \quad \forall y \in \mathscr{B}_{1} .
$$

That is why the name of this stability is Ulam-Hyers stability. Later on, Hyers results are extended by many mathematicians; for details, reader may see [32-39] and the reference therein. The mentioned stability analysis is extremely helpful in numerous applications, for example, numerical analysis and optimization, where it is very tough to find the exact solution of a nonlinear problem. We notice that Ulam-Hyers stability concept is quite significant in realistic problems in numerical analysis, biology, and economics. The aforementioned stability has very recently attracted the attention of researchers; we refer the reader to some papers in [4045]. Because fractional order system may have additional attractive feature over the integer order system, let us suppose the following example to show which one is more stable in the aforementioned (fractional order and integer order) systems.

Example (see [46]). We have the following two systems with initial condition $y(0)$ for $v \in(0,1)$,

$$
\begin{aligned}
\frac{d}{d t} y(t) & =v t^{v-1}, \\
{ }_{0}^{c} D_{t}^{\rho} y(t) & =v t^{v-1}, \quad 0<\rho<1 .
\end{aligned}
$$

Then the analytical solutions of (5) and (6) are $t^{v}+y(0)$ and $v \Gamma(v) t^{v+\rho-1} / \Gamma(v+\rho)+y(0)$, respectively. Clearly, the integer order system (5) is unstable for any $0<v<1$. But the fractional dynamic system (6) is stable for each $0<v \leq$ $1-\rho$. So fractional order system may have better features than integer order system.

Influenced from the above-mentioned work, we will study the existence, uniqueness, and different types of Ulam-Hyers stability of the following implicit impulsive fractional order differential equations with nonlocal boundary conditions, involving Caputo derivative

$$
\begin{aligned}
{ }^{c} D^{\rho} y(t) & =f\left(t, y(t),{ }^{c} D^{\rho} y(t)\right), \\
t \in \Im=[0,1], t \neq t_{j}, j=1,2, \ldots, k, & \\
\Delta y\left(t_{j}\right) & =I_{j}\left(y\left(t_{j}\right)\right), \\
\Delta y^{\prime}\left(t_{j}\right) & =\widehat{I}_{j}\left(y\left(t_{j}\right)\right), \\
y(0) & =g(y), \\
y(1) & =h(y),
\end{aligned}
$$

where $1<\rho \leq 2, f: \mathfrak{I} \times \mathbb{R} \times \mathbb{R} \rightarrow \mathbb{R}$ is continuous, $I_{j}, \widehat{I}_{j}$ : $\mathbb{R} \rightarrow \mathbb{R}$ are continuous, and

$$
\begin{gathered}
\Delta\left(y\left(t_{j}\right)\right)=y\left(t_{j}^{+}\right)-y\left(t_{j}^{-}\right), \\
\Delta\left(y^{\prime}\left(t_{j}\right)\right)=y^{\prime}\left(t_{j}^{+}\right)-y^{\prime}\left(t_{j}^{-}\right)
\end{gathered}
$$

with $y\left(t_{j}^{+}\right), y^{\prime}\left(t_{j}^{+}\right), y\left(t_{j}^{-}\right), y^{\prime}\left(t_{j}^{-}\right)$are the respective left and right limits of $y\left(t_{j}\right)$ at $t=t_{j}$. 
The manuscript is structured as follows. In Section 2, we give some definitions, theorems, lemmas, and remarks. In Section 3, we built up some adequate conditions for the existence and uniqueness of solutions to the considered problem (7) through using fixed point theorems of Krasnoselski's and Banach contraction type. In Section 4, we establish applicable results under which the solution of the considered boundary value problem (7) satisfies the conditions of different kinds of Ulam-Hyers stability. The established results are illustrated by an example in Section 5 .

\section{Background Materials and Auxiliary Results}

This section is devoted to some basic definitions, theorems, lemmas, and remarks which are useful in existence and stability results.

Definition 1 (see [11]). For a function $y \in((0, \infty), \mathbb{R})$, the Caputo derivative of fractional order $\rho \in \mathbb{R}^{+}$is defined as

$$
\begin{array}{r}
{ }^{c} D^{\rho} y(t)=\frac{1}{\Gamma(n-\rho)} \int_{0}^{t}(t-\kappa){ }^{n-\rho-1} y^{(n)}(\kappa) d \kappa, \\
n=[\rho]+1,
\end{array}
$$

where $[\rho]$ denotes the integer part of $\rho$.

Definition 2 (see [11]). The fractional integral of order $\rho \in \mathbb{R}^{+}$ of function $y \in L^{1}\left(\mathfrak{I}, \mathbb{R}^{+}\right)$is given by

$$
I^{\rho} y(t)=\frac{1}{\Gamma(\rho)} \int_{0}^{t}(t-\kappa)^{\rho-1} y(\kappa) d \kappa,
$$

where

$$
\Gamma(\rho)=\int_{0}^{\infty} t^{\rho-1} e^{-t} d t, \quad \rho>0 .
$$

Lemma 3 (see [11]). Let $\rho>0$, then the differential equations

$$
{ }^{c} D^{\rho} y(t)=0
$$

has solution given by

$$
\begin{aligned}
y(t)=c_{0}+c_{1} t+c_{2} t^{2}+\cdots+c_{n-1} t^{n-1}, & \\
& c_{i} \in \mathbb{R}, i=0,1,2, \ldots, n-1,
\end{aligned}
$$

where $n=[\rho]+1$.

Lemma 4 (see [11]). Let $\rho>0$, then the solution of the differential equation

$$
{ }^{c} D^{\rho} y(t)=x(t)
$$

will be in the following form:

$$
\begin{aligned}
& I^{\rho}\left[D^{\rho} y(t)\right]=I^{\rho} x(t)+c_{0}+c_{1} t+c_{2} t^{2}+\cdots \\
& +c_{n-1} t^{n-1}, \\
& \quad c_{i} \in \mathbb{R}, \quad i=0,1,2, \ldots, n-1,
\end{aligned}
$$

where $n=[\rho]+1$.

Lemma 5. For $t_{j} \in(0,1)$ such that $t_{1}<t_{2}<\cdots<t_{j}$, let $\mathfrak{I}^{\prime}=\mathfrak{I}-\left\{t_{1}, t_{2}, \ldots, t_{j}\right\}$ define the space

$$
\mathbb{X}=\left\{y: \mathfrak{J} \longrightarrow \mathbb{R}: y \in C\left(\mathfrak{I}^{\prime}\right) \text { and left } y\left(t_{j}^{+}\right) \text {and right limit } y\left(t_{j}^{-}\right) \text {exist and } y\left(t_{j}^{-}\right)=y\left(t_{j}\right), j \in[1, k]\right\} \text {. }
$$

Then, clearly $\mathbb{X}=C^{2}(\mathfrak{I}, \mathbb{R})$ is Banach space endowed with a norm $\|y\|=\max _{t \in \mathfrak{I}}|y(t)|$.

Theorem 6 ((Krasnoselskii's fixed point theorem) see [47]). Consider $\mathbb{U}$ be a convex closed and nonempty subset of Banach space $\mathbb{X}$. Suppose $\mathscr{F}^{*}, \mathscr{G}^{*}$ be the operators such that

(i) $\mathscr{F}^{*} x+\mathscr{G}^{*} y \in \mathbb{U}$ whenever $x, y \in \mathbb{U}$;

(ii) $\mathscr{F}^{*}$ is compact and continuous and $\mathscr{G}^{*}$ is contraction mapping.

Then there is $w \in \mathbb{U}$ such that $w=\mathscr{F}^{*} w+\mathscr{G}^{*} w$.

Theorem 7 ((Banach fixed point theorem) see [47]). Suppose $\mathcal{S}$ be a nonempty closed subset of a Banach space $\mathscr{B}$. Then any contraction mapping $\mathfrak{D}$ from $\mathscr{B}$ into itself has a unique fixed point.

Ulam Type Stability. Let $z \in \mathbb{X}$ and $\epsilon, \wp$ be positive real numbers and $\varphi \in C\left(\mathfrak{I}, \mathbb{R}^{+}\right)$a nondecreasing function. Then the following inequalities exist:

$$
\begin{aligned}
& \left|{ }^{c} D^{\rho} z(t)-f\left(t, z(t),{ }^{c} D^{\rho} z(t)\right)\right| \leq \epsilon, \\
& t \in \mathfrak{\Im}_{j}, \quad j=1,2, \ldots, k ; \\
& \left|\Delta z\left(t_{j}\right)-I_{j}\left(z\left(t_{j}\right)\right)\right| \leq \epsilon, \\
& j=1,2, \ldots, k \text {; } \\
& \left|\Delta z^{\prime}\left(t_{j}\right)-\widehat{I}_{j}\left(z\left(t_{j}\right)\right)\right| \leq \epsilon, \\
& j=1,2, \ldots, k, \\
& \left|{ }^{c} D^{\rho} z(t)-f\left(t, z(t),{ }^{c} D^{\rho} z(t)\right)\right| \leq \varphi(t), \\
& t \in \mathfrak{J}_{j}, j=1,2, \ldots, k ; \\
& \left|\Delta z\left(t_{j}\right)-I_{j}\left(z\left(t_{j}\right)\right)\right| \leq \wp, \\
& j=1,2, \ldots, k \text {; } \\
& \left|\Delta z^{\prime}\left(t_{j}\right)-\widehat{I}_{j}\left(z\left(t_{j}\right)\right)\right| \leq \wp,
\end{aligned}
$$




$$
\begin{aligned}
\left|{ }^{c} D^{\rho} z(t)-f\left(t, z(t),{ }^{c} D^{\rho} z(t)\right)\right| & \leq \varphi(t) \epsilon, \\
t & \in \Im_{j}, j=1,2, \ldots, k ; \\
\left|\Delta z\left(t_{j}\right)-I_{j}\left(z\left(t_{j}\right)\right)\right| \leq \wp \epsilon, & \\
j=1,2, \ldots, k ; & j=1,2, \ldots, k .
\end{aligned}
$$

Definition 8 (see [48]). The considered problem (7) is Ulam-Hyers stable, if there is a constant $c_{f, k} \in \mathbb{R}^{+}$such that, for every $\epsilon>0$ and for every solution $z \in \mathbb{X}$ of inequality (17), there is a unique solution $y \in \mathbb{X}$ of the considered problem (7) with

$$
|z(t)-y(t)| \leq c_{f, k} \epsilon, \quad t \in \mathfrak{\Im} .
$$

Definition 9 (see [48]). The considered problem (7) is generalized Ulam-Hyers stable, if there is $\phi_{f, k} \in C\left(\mathbb{R}^{+}, \mathbb{R}^{+}\right)$, $\phi_{f, k}(0)=0$, such that, for every solution $z \in \mathbb{X}$ of inequality (17), there is a unique solution $y \in \mathbb{X}$ of the considered problem (7) with

$$
|z(t)-y(t)| \leq \varphi_{f, k}(\epsilon), \quad t \in \mathfrak{\Im} .
$$

Definition 10 (see [48]). The considered problem (7) is Ulam-Hyers-Rassias stable with respect to $\varphi \in C\left(\mathfrak{I}, \mathbb{R}^{+}\right)$, if there is a constant $c_{f, k, \varphi} \in \mathbb{R}^{+}$, such that, for every $\epsilon>0$ and for every solution $z \in \mathbb{X}$ of inequality (18), there is a unique solution $y \in \mathbb{X}$ of the considered problem (7) with

$$
|z(t)-y(t)| \leq c_{f, k, \varphi} \epsilon \varphi(t), \quad t \in \mathfrak{J} .
$$

Definition 11 (see [48]). The considered problem (7) is generalized Ulam-Hyers-Rassias stable with respect to $\varphi \in$ $C\left(\mathfrak{I}, \mathbb{R}^{+}\right)$, if there is a constant $c_{f, k, \varphi} \in \mathbb{R}^{+}$, such that, for every solution $z \in \mathbb{X}$ of inequality (19), there is a unique solution $y \in \mathbb{X}$ of the considered problem (7) with

$$
|z(t)-y(t)| \leq c_{f, k, \varphi} \varphi(t), \quad t \in \mathfrak{J} .
$$

Remark 12. A function $z \in \mathbb{X}$ is a solution of inequality (17), if there is a function $\vartheta \in \mathbb{X}$ and a sequence $\vartheta_{j}, j=1,2, \ldots, k$ (which depend on $z$ only), such that

(i) $|\vartheta(t)| \leq \epsilon,\left|\vartheta_{j}\right| \leq \epsilon, t \in \Im_{j}, j=1,2, \ldots, k$;

(ii) ${ }^{c} D^{\rho} z(t)=f\left(t, z(t),{ }^{c} D^{\rho} z(t)\right)+\vartheta(t), t \in \mathfrak{\Im}_{j}, j=$ $1,2, \ldots, k$

(iii) $\Delta y\left(t_{j}\right)=I_{j}\left(y\left(t_{j}\right)\right)+\vartheta_{j}, t \in \mathfrak{J}_{j}, j=1,2, \ldots, k$;

(iv) $\Delta y^{\prime}\left(t_{j}\right)=\widehat{I}_{j}\left(y\left(t_{j}\right)\right)+\vartheta_{j}, t \in \mathfrak{\Im}_{j}, j=1,2, \ldots, k$.

Remark 13. A function $z \in \mathbb{X}$ is a solution of inequality (18), if there is a function $\vartheta \in \mathbb{X}$ and a sequence $\vartheta_{j}, j=1,2, \ldots, k$ (which depend on $z$ only), such that

(i) $|\vartheta(t)| \leq \varphi(t),\left|\vartheta_{j}\right| \leq \wp, t \in \mathfrak{J}_{j}, j=1,2, \ldots, k$;

(ii) ${ }^{c} D^{\rho} z(t)=f\left(t, z(t),{ }^{c} D^{\rho} z(t)\right)+\vartheta(t), t \in \Im_{j}, j=$ $1,2, \ldots, k$

$$
\begin{aligned}
& \text { (iii) } \Delta y\left(t_{j}\right)=I_{j}\left(y\left(t_{j}\right)\right)+\vartheta_{j}, t \in \mathfrak{\Im}_{j}, j=1,2, \ldots, k \text {; } \\
& \text { (iv) } \Delta y^{\prime}\left(t_{j}\right)=\widehat{I}_{j}\left(y\left(t_{j}\right)\right)+\vartheta_{j}, t \in \mathfrak{\Im}_{j}, j=1,2, \ldots, k .
\end{aligned}
$$

Remark 14. A function $z \in \mathbb{X}$ is a solution of inequality (19), if there is a function $\vartheta \in \mathbb{X}$ and a sequence $\vartheta_{j}, j=1,2, \ldots, k$ (which depend on $z$ only), such that

(i) $|\vartheta(t)| \leq \varphi(t) \epsilon,\left|\vartheta_{j}\right| \leq \wp \epsilon, t \in \mathfrak{J}_{j}, j=1,2, \ldots, k$;

(ii) ${ }^{c} D^{\rho} z(t)=f\left(t, z(t),{ }^{c} D^{\rho} z(t)\right)+\mathcal{\vartheta}(t), t \in \Im_{j}, j=$ $1,2, \ldots, k$

(iii) $\Delta y\left(t_{j}\right)=I_{j}\left(y\left(t_{j}\right)\right)+\vartheta_{j}, t \in \Im_{j}, j=1,2, \ldots, k$;

(iv) $\Delta y^{\prime}\left(t_{j}\right)=\widehat{I}_{j}\left(y\left(t_{j}\right)\right)+\vartheta_{j}, t \in \Im_{j}, j=1,2, \ldots, k$.

\section{Main Results}

Theorem 15. For $x \in C(\mathfrak{I}, \mathbb{R})$, the following linear boundary value problem

$$
\begin{aligned}
{ }^{c} D^{\rho} y(t) & =x(t), \\
& t \in \mathfrak{J}, t \neq t_{j}, j=1,2, \ldots, k, \rho \in(1,2], \\
\Delta y\left(t_{j}\right) & =I_{j}\left(y\left(t_{j}\right)\right), \\
\Delta y^{\prime}\left(t_{j}\right) & =\widehat{I}_{j}\left(y\left(t_{j}\right)\right), \\
y(0) & =h(y), \\
y(1) & =g(y)
\end{aligned}
$$

has a solution of the form

$$
\begin{aligned}
y(t) & \\
= & t g(y)+(1-t) h(y)+\sum_{j=1}^{k}\left(t-t_{j}\right) \widehat{I}_{j}\left(y\left(t_{j}\right)\right) \\
& -\sum_{j=1}^{k} t\left(1-t_{j}\right) \widehat{I}_{j} y\left(t_{j}\right)+\sum_{j=1}^{k} I_{j}\left(y\left(t_{j}\right)\right) \\
& -\sum_{j=1}^{k} t I_{j} y\left(t_{j}\right)+\frac{1}{\Gamma(\rho)} \int_{t_{j}}^{t}(t-\kappa)^{\rho-1} x(\kappa) d \kappa \\
& +\frac{1}{\Gamma(\rho)} \sum_{j=1}^{k} \int_{t_{j-1}}^{t_{j}}\left(t_{j}-\kappa\right)^{\rho-1} x(\kappa) d \kappa \\
& +\frac{1}{\Gamma(\rho-1)} \sum_{j=1}^{k}\left(t-t_{j}\right) \int_{t_{j-1}}^{t_{j}}\left(t_{j}-\kappa\right)^{\rho-2} x(\kappa) d \kappa \\
& -\frac{t}{\Gamma(\rho)} \sum_{j=1}^{k+1} \int_{t_{j-1}}^{t_{j}}\left(t_{j}-\kappa\right)^{\rho-1} x(\kappa) d \kappa \\
& k \sum_{j=1}^{k}\left(1-t_{j}\right) \int_{t_{j-1}}^{t_{j}}\left(t_{j}-\kappa\right)^{\rho-2} x(\kappa) d \kappa .
\end{aligned}
$$


Proof. Apply Lemma 4 to the differential equation (24) with using constants $c_{0}, c_{1} \in \mathbb{R}$, such that

$$
\begin{aligned}
y(t) & =I^{\rho} x(t)-c_{0}-c_{1} t \\
& =\frac{1}{\Gamma(\rho)} \int_{0}^{t}(t-\kappa)^{\rho-1} x(\kappa) d \kappa-c_{0}-c_{1} t,
\end{aligned}
$$

$$
t \in\left[0, t_{1}\right]
$$

and also

$$
\begin{aligned}
y^{\prime}(t) & =I^{\rho-1} x(t)-c_{1} \\
& =\frac{1}{\Gamma(\rho-1)} \int_{0}^{t}(t-\kappa)^{\rho-2} x(\kappa) d \kappa-c_{1}, \\
& \quad t \in\left[0, t_{1}\right] .
\end{aligned}
$$

$$
y^{\prime}(t)=\frac{1}{\Gamma(\rho-1)} \int_{t_{1}}^{t}(t-\kappa)^{\rho-2} x(\kappa) d \kappa-d_{1} .
$$

Hence, it follows that

$$
\begin{aligned}
& y\left(t_{1}^{-}\right)=\frac{1}{\Gamma(\rho)} \int_{0}^{t_{1}}\left(t_{1}-\kappa\right)^{\rho-1} x(\kappa) d \kappa-c_{0}-c_{1} t_{1}, \\
& y\left(t_{1}^{+}\right)=-d_{0}, \\
& y^{\prime}\left(t_{1}^{-}\right)=\frac{1}{\Gamma(\rho-1)} \int_{0}^{t_{1}}\left(t_{1}-\kappa\right)^{\rho-2} x(\kappa) d \kappa-c_{1}, \\
& y^{\prime}\left(t_{1}^{+}\right)=-d_{1} .
\end{aligned}
$$

Using the following impulsive conditions

$$
\begin{gathered}
\Delta y\left(t_{1}\right)=y\left(t_{1}^{+}\right)-y\left(t_{1}^{-}\right)=I_{1}\left(y\left(t_{1}\right)\right), \\
\Delta y^{\prime}\left(t_{1}\right)=y^{\prime}\left(t_{1}^{+}\right)-y^{\prime}\left(t_{1}^{-}\right)=\widehat{I}_{1}\left(y\left(t_{1}\right)\right) .
\end{gathered}
$$

We obtain

$$
\begin{aligned}
-d_{0}= & \frac{1}{\Gamma(\rho)} \int_{0}^{t_{1}}\left(t_{1}-\kappa\right)^{\rho-1} x(\kappa) d \kappa-c_{0}-c_{1} t_{1} \\
& +I_{1}\left(y\left(t_{1}\right)\right), \\
-d_{1}= & \frac{1}{\Gamma(\rho-1)} \int_{0}^{t_{1}}\left(t_{1}-\kappa\right)^{\rho-2} x(\kappa) d \kappa-c_{1} \\
& +\widehat{I}_{1}\left(y\left(t_{1}\right)\right) .
\end{aligned}
$$

Hence, we get

$$
\begin{aligned}
y(t)= & \frac{1}{\Gamma(\rho)} \int_{t_{1}}^{t}(t-\kappa)^{\rho-1} x(\kappa) d \kappa \\
& +\frac{1}{\Gamma(\rho)} \int_{0}^{t_{1}}\left(t_{1}-\kappa\right)^{\rho-1} x(\kappa) d \kappa
\end{aligned}
$$

$$
\begin{aligned}
& +\frac{t-t_{1}}{\Gamma(\rho-1)} \int_{0}^{t_{1}}\left(t_{1}-\kappa\right)^{\rho-2} x(\kappa) d \kappa \\
& +\left(t-t_{1}\right) \widehat{I}_{1}\left(y\left(t_{1}\right)\right)+I_{1}\left(y\left(t_{1}\right)\right)-c_{0}-c_{1} t, \\
& t \in\left(t_{1}, t_{2}\right] .
\end{aligned}
$$

Going on the similar way, we get the following expression for the solution $y(t)$ for $t \in\left(t_{j}, t_{j+1}\right]$ :

$y(t)$

$$
\begin{aligned}
= & \frac{1}{\Gamma(\rho)} \int_{t_{j}}^{t}(t-\kappa)^{\rho-1} x(\kappa) d \kappa \\
& +\frac{1}{\Gamma(\rho)} \sum_{j=1}^{k} \int_{t_{j-1}}^{t_{j}}\left(t_{j}-\kappa\right)^{\rho-1} x(\kappa) d \kappa \\
& +\frac{1}{\Gamma(\rho-1)} \sum_{j=1}^{k}\left(t-t_{j}\right) \int_{t_{j-1}}^{t_{j}}\left(t_{j}-\kappa\right)^{\rho-2} x(\kappa) d \kappa \\
& +\sum_{j=1}^{k}\left(t-t_{j}\right) \widehat{I}_{j}\left(y\left(t_{j}\right)\right)+\sum_{j=1}^{k} I_{j}\left(y\left(t_{j}\right)\right)-c_{0} \\
& -c_{1} t, \quad t \in\left(t_{j}, t_{j+1}\right], j=1,2, \ldots, k .
\end{aligned}
$$

Now, by applying the nonlocal boundary conditions $y(0)=$ $g(y), y(1)=h(y)$ on (33) and calculating the values of $c_{0}, c_{1}$, (25) can be obtained.

Conversely, if $y(t)$ is the solution of integral equation (25), then it is obvious that ${ }^{c} D^{\rho} y(t)=x(t)$ and $y(0)=g(y), y(1)=$ $h(y), \Delta y\left(t_{j}\right)=I_{j}\left(y\left(t_{j}\right)\right), \Delta y^{\prime}\left(t_{j}\right)=\widehat{I}_{j}\left(y\left(t_{j}\right)\right), j=1,2, \ldots, k$. Hence proof is completed.

We define $\mathfrak{D}: \mathbb{X} \rightarrow \mathbb{X}$ by

D) $y(t)$

$$
\begin{aligned}
& =\operatorname{tg}(y)+(1-t) h(y)+\sum_{j=1}^{k}\left(t-t_{j}\right) \widehat{I}_{j}\left(y\left(t_{j}\right)\right) \\
& -\sum_{j=1}^{k} t\left(1-t_{j}\right) \widehat{I}_{j} y\left(t_{j}\right)+\sum_{j=1}^{k} I_{j}\left(y\left(t_{j}\right)\right) \\
& -\sum_{j=1}^{k} t I_{j} y\left(t_{j}\right)+\frac{1}{\Gamma(\rho)} \int_{t_{j}}^{t}(t-\kappa)^{\rho-1} u(\kappa) d \kappa \\
& +\frac{1}{\Gamma(\rho)} \sum_{j=1}^{k} \int_{t_{j-1}}^{t_{j}}\left(t_{j}-\kappa\right)^{\rho-1} u(\kappa) d \kappa \\
& +\frac{1}{\Gamma(\rho-1)} \sum_{j=1}^{k}\left(t-t_{j}\right) \int_{t_{j-1}}^{t_{j}}\left(t_{j}-\kappa\right)^{\rho-2} u(\kappa) d \kappa
\end{aligned}
$$




$$
\begin{aligned}
& -\frac{t}{\Gamma(\rho)} \sum_{j=1}^{k+1} \int_{t_{j-1}}^{t_{j}}\left(t_{j}-\kappa\right)^{\rho-1} u(\kappa) d \kappa \\
& -\frac{t}{\Gamma(\rho-1)} \sum_{j=1}^{k}\left(1-t_{j}\right) \int_{t_{j-1}}^{t_{j}}\left(t_{j}-\kappa\right)^{\rho-2} u(\kappa) d \kappa,
\end{aligned}
$$

where

$$
\begin{aligned}
& u(t)=f(t, y(t), u(t)), \\
& u(t)={ }^{c} D^{\rho} y(t) .
\end{aligned}
$$

Suppose that the following hold.

$\left(A_{1}\right)$ For any $y, z \in \mathbb{X}$, there exist $K_{g}, K_{h}$, such that

$$
\begin{gathered}
\|g(y)-g(z)\| \leq K_{g}\|y-z\|, \\
\|h(y)-h(z)\| \leq K_{h}\|y-z\| .
\end{gathered}
$$

$\left(A_{2}\right)$ For any $y, z, x, w \in \mathbb{R}$ and for each $t \in \mathfrak{I}$, there exist $L_{f}>0,0<K_{f}<1$ such that

$$
|f(t, y, x)-f(t, z, w)| \leq L_{f}|y-z|+K_{f}|x-w| .
$$

$\left(A_{3}\right)$ For $y, z \in \mathbb{R}$, there exist constants $\mathbb{A}, \mathbb{B}>0$ such that

$$
\begin{aligned}
& \left|I_{j}(y)-I_{j}(z)\right| \leq \mathbb{A}|y-z|, \\
& \left|\widehat{I}_{j}(y)-\widehat{I}_{j}(z)\right| \leq \mathbb{B}|y-z|,
\end{aligned}
$$

$$
j=1,2, \ldots, k \text {. }
$$

The following result is based on Banach contraction theorem.

Theorem 16. Under the assumptions $\left(A_{1}\right)-\left(A_{3}\right)$ and if

$$
\frac{(4 k+2) L_{f}}{\left(1-K_{f}\right) \Gamma(\rho)}+K_{g}+K_{h}+k(\mathbb{A}+\mathbb{B})<1,
$$

the considered problem (7) has a unique positive solution.

Proof. Suppose $y, z \in \mathbb{X}$ and for every $t \in \mathfrak{I}$, consider

$$
\begin{aligned}
& |\mathfrak{D} y(t)-\mathfrak{D} z(t)|=|y(t)-z(t)| \leq|\operatorname{tg}(y)-\operatorname{tg}(z)| \\
& +|(1-t)[h(y)-h(z)]| \\
& +\mid \sum_{j=1}^{k}\left[\left(t-t_{j}\right)+t\left(1-t_{j}\right)\right] \\
& \cdot\left[\widehat{I}_{j}\left(y\left(t_{j}\right)\right)-\widehat{I}_{j}\left(z\left(t_{j}\right)\right)\right]|+| \sum_{j=1}^{k}(1-t) \\
& \cdot\left[I_{j}\left(y\left(t_{j}\right)\right)-I_{j} z\left(t_{j}\right)\right]|+| \frac{1}{\Gamma(\rho)} \int_{t_{j}}^{t}(t-\kappa)^{\rho-1}
\end{aligned}
$$

$$
\begin{aligned}
& \cdot[u(\kappa)-\bar{u}(\kappa)] d \kappa|+| \frac{1}{\Gamma(\rho)} \\
& \cdot \sum_{j=1}^{k} \int_{t_{j-1}}^{t_{j}}\left(t_{j}-\kappa\right)^{\rho-1}[u(\kappa)-\bar{u}(\kappa)] d \kappa|+| \frac{t}{\Gamma(\rho)} \\
& \cdot \sum_{j=1}^{k+1} \int_{t_{j-1}}^{t_{j}}\left(t_{j}-\kappa\right)^{\rho-1}[u(\kappa)-\bar{u}(\kappa)] d \kappa \mid \\
& +\mid \frac{1}{\Gamma(\rho-1)} \sum_{j=1}^{k}\left(t-t_{j}\right) \int_{t_{j-1}}^{t_{j}}\left(t_{j}-\kappa\right)^{\rho-2}
\end{aligned}
$$$$
\cdot[u(\kappa)-\bar{u}(\kappa)] d \kappa|+| \frac{t}{\Gamma(\rho-1)} \sum_{j=1}^{k}\left(1-t_{j}\right)
$$$$
\cdot \int_{t_{j-1}}^{t_{j}}\left(t_{j}-\kappa\right)^{\rho-2}[u(\kappa)-\bar{u}(\kappa)] d \kappa \mid \text {, }
$$

where

$$
\begin{aligned}
& u(t)=f(t, y(t), u(t)) \\
& \bar{u}(t)=f(t, z(t), \bar{u}(t))
\end{aligned}
$$

So

$$
\begin{aligned}
\|\mathfrak{D} y-\mathfrak{D} z\| \leq & K_{g}\|y-z\|+K_{h}\|y-z\| \\
& +k(\mathbb{A}+\mathbb{B})\|y-z\| \\
& +\frac{L_{f}}{\left(1-K_{f}\right) \Gamma(\rho+1)}\|y-z\| \\
& +\frac{k L_{f}}{\left(1-K_{f}\right) \Gamma(\rho+1)}\|y-z\| \\
& +\frac{k L_{f}}{\left(1-K_{f}\right) \Gamma(\rho)}\|y-z\| \\
& +\frac{(k+1) L_{f}}{\left(1-K_{f}\right) \Gamma(\rho+1)}\|y-z\| \\
& +\frac{k L_{f}}{\left(1-K_{f}\right) \Gamma(\rho)}\|y-z\|,
\end{aligned}
$$

which implies that

$$
\begin{array}{rl}
\| \mathfrak{D} & y-\mathfrak{D} z \| \\
\leq & {\left[\frac{(4 k+2) L_{f}}{\left(1-K_{f}\right) \Gamma(\rho)}+K_{g}+K_{h}+k(\mathbb{A}+\mathbb{B})\right]} \\
& \cdot\|y-z\|,
\end{array}
$$

which implies that $\mathfrak{D}$ is contraction. Hence, the considered problem (7) has a unique positive solution. 
The following result is based on Krasnoselskii's fixed point theorem.

Theorem 17. In addition to assumptions $\left(A_{1}\right)-\left(A_{3}\right)$, let the following hold.

$\left(A_{4}\right)$ There exist constants $\omega, \mu$ such that

$$
\begin{aligned}
& |g(y)| \leq \omega, \\
& |h(y)| \leq \mu
\end{aligned}
$$

$$
\forall y \in \mathbb{X} .
$$

$\left(A_{5}\right)$ For $t \in \mathfrak{J}$, there exist $n, o, p \in C\left(\mathfrak{J}, \mathbb{R}^{+}\right)$, such that

$$
|f(t, y(t), u(t))| \leq n(t)+o(t)|y(t)|+p(t)|u(t)|
$$$$
\text { for } y, w \in \mathbb{R} \text {. }
$$

with $n^{*}=\sup _{t \in \mathfrak{J}^{n}}(t), o^{*}=\sup _{t \in \mathfrak{J}^{o}}(t)$, and $p^{*}=$ $\sup _{t \in \mathfrak{I}} p(t)<1$.

$\left(A_{6}\right)$ The function $I_{j}: \mathbb{R} \rightarrow \mathbb{R}$ is continuous and there exist constants $\mathbb{C}^{*}, \mathbb{D}^{*}>0$ such that $j=1,2, \ldots, k$,

$\left|I_{j}\left(y_{j}\right)\right| \leq \mathbb{C}^{*}|y|+\mathbb{D}^{*} \quad$ for each $y \in(\mathfrak{J}, R)$,

$\left(A_{7}\right)$ The function $\widehat{I}_{j}: \mathbb{R} \rightarrow \mathbb{R}$ is continuous and there exist constants $\mathbb{E}^{*}, \mathbb{F}^{*}>0$ such that $j=1,2, \ldots, k$,

$$
\left|\widehat{I}_{j}\left(y_{j}\right)\right| \leq \mathbb{E}^{*}|y|+\mathbb{F}^{*} \quad \text { for each } y \in(\mathfrak{I}, R) \text {. }
$$

If

$$
K_{g}+K_{h}+k(\mathbb{A}+\mathbb{B})<1,
$$

then the considered problem (7) has at least one positive solution.

Proof. Take $|f(t, y(t), u(t))| \leq n(t)+o(t)|y(t)|+p(t)|u(t)|$ for $y, u \in \mathbb{R}$, with $\|y\| \leq \xi^{*}$, where $\xi^{*}>0$, such that

$$
\begin{aligned}
\mathscr{R} \geq & \omega+\mu+k\left(\left(\mathbb{E}^{*}+\mathbb{C}^{*}\right) \xi^{*}+\left(\mathbb{F}^{*}+\mathbb{D}^{*}\right)\right) \\
& +\frac{(4 k+2) \mathcal{N}_{0}}{\Gamma(\rho)} .
\end{aligned}
$$

Construct a closed ball $\mathcal{S} \subset \mathbb{X}$, such that

$$
y \in \mathcal{S}=\{y \in \mathbb{X}:\|y\| \leq \mathscr{R}\} .
$$

Split the operator $\mathfrak{D}$ into two parts as $\mathfrak{D}=\mathscr{F}^{*}+\mathscr{G}^{*}$, where

$$
\begin{gathered}
\mathscr{F}^{*} y(t)=\frac{1}{\Gamma(\rho)} \int_{t_{j}}^{t}(t-\kappa)^{\rho-1} f(\kappa, y(\kappa), u(\kappa)) d \kappa \\
+\frac{1}{\Gamma(\rho)} \sum_{j=1}^{k} \int_{t_{j-1}}^{t_{j}}\left(t_{j}-\kappa\right)^{\rho-1} f(\kappa, y(\kappa), u(\kappa)) d \kappa \\
+\frac{1}{\Gamma(\rho-1)} \sum_{j=1}^{k}\left(t-t_{j}\right)
\end{gathered}
$$

$$
\begin{aligned}
& \cdot \int_{t_{j-1}}^{t_{j}}\left(t_{j}-\kappa\right)^{\rho-2} f(\kappa, y(\kappa), u(\kappa)) d \kappa-\frac{t}{\Gamma(\rho)} \\
& \cdot \sum_{j=1}^{k+1} \int_{t_{j-1}}^{t_{j}}\left(t_{j}-\kappa\right)^{\rho-1} f(\kappa, y(\kappa), u(\kappa)) d \kappa \\
& -\frac{t}{\Gamma(\rho-1)} \sum_{j=1}^{k}\left(1-t_{j}\right) \\
& \cdot \int_{t_{j-1}}^{t_{j}}\left(t_{j}-\kappa\right)^{\rho-2} f(\kappa, y(\kappa), u(\kappa)) d \kappa \\
& \mathscr{G}^{*} y^{(t)}=t g(y)+(1-t) h(y) \\
& +\sum_{j=1}^{k}\left(t-t_{j}\right) \widehat{I}_{j}\left(y\left(t_{j}\right)\right)-\sum_{j=1}^{k} t\left(1-t_{j}\right) \widehat{I}_{j} y\left(t_{j}\right) \\
& +\sum_{j=1}^{k} I_{j}\left(y\left(t_{j}\right)\right)-\sum_{j=1}^{k} t I_{j} y\left(t_{j}\right) .
\end{aligned}
$$

Clearly $\mathfrak{D}=\mathscr{F}^{*}+\mathscr{G}^{*}$. Now we show that $\mathfrak{D} y=\mathscr{F}^{*} y+\mathscr{G}^{*} y \in$ $\mathcal{S}$, for all $y \in \mathcal{S}$. For any $y \in \mathcal{S}$, we have

$$
\begin{aligned}
& |\mathfrak{D} y(t)|=\mid \frac{1}{\Gamma(\rho)} \int_{t_{j}}^{t}(t-\kappa)^{\rho-1} u(\kappa) d \kappa \\
& +\frac{1}{\Gamma(\rho)} \sum_{j=1}^{k} \int_{t_{j-1}}^{t_{j}}\left(t_{j}-\kappa\right)^{\rho-1} u(\kappa) d \kappa \\
& +\frac{1}{\Gamma(\rho-1)} \sum_{j=1}^{k}\left(t-t_{j}\right) \int_{t_{j-1}}^{t_{j}}\left(t_{j}-\kappa\right)^{\rho-2} u(\kappa) d \kappa \\
& -\frac{t}{\Gamma(\rho)} \sum_{j=1}^{k+1} \int_{t_{j-1}}^{t_{j}}\left(t_{j}-\kappa\right)^{\rho-1} u(\kappa) d \kappa \\
& -\frac{t}{\Gamma(\rho-1)} \sum_{j=1}^{k}\left(1-t_{j}\right) \int_{t_{j-1}}^{t_{j}}\left(t_{j}-\kappa\right)^{\rho-2} u(\kappa) d \kappa \\
& +t g(y)+(1-t) h(y)+\sum_{j=1}^{k}\left(t-t_{j}\right) \widehat{I}_{j}\left(y\left(t_{j}\right)\right) \\
& -\sum_{j=1}^{k} t I_{j} y\left(t_{j}\right) \mid, \\
& -\sum_{j=1}^{k} t\left(1-t_{j}\right) \widehat{I}_{j} y\left(t_{j}\right)+\sum_{j=1}^{k} I_{j}\left(y\left(t_{j}\right)\right)
\end{aligned}
$$

where

$$
u(t)=f(t, y(t), u(t))
$$


8

Complexity

Now by $\left(A_{5}\right)$, we have

$$
\begin{aligned}
|u(t)| & =|f(t, y(t), u(t))| \\
& \leq n(t)+o(t)|y(t)|+p(t)|u(t)| \\
& \leq n(t)+o(t) \xi^{*}+p(t)|u(t)| \\
& \leq n^{*}+o^{*} \xi^{*}+p^{*}\|u\| .
\end{aligned}
$$

Therefore, we get

$$
\|u\| \leq \frac{n^{*}+o^{*} \xi^{*}}{1-p^{*}}=\mathcal{N}_{0} .
$$

Using $\left(A_{4}\right),\left(A_{6}\right),\left(A_{7}\right)$ and (55) in (52), we get

$$
\begin{aligned}
\|\mathfrak{D} y\| \leq & \omega+\mu+k\left(\left(\mathbb{E}^{*}+\mathbb{C}^{*}\right) \xi^{*}+\left(\mathbb{F}^{*}+\mathbb{D}^{*}\right)\right) \\
& +\frac{(4 k+2) \mathscr{N}_{0}}{\Gamma(\rho)} \leq \mathscr{R} .
\end{aligned}
$$

Hence, we get that $|\mathfrak{D} y(t)| \leq \mathscr{R}$ which implies that $\mathfrak{D}(\mathcal{S}) \subseteq$ $\mathcal{S}$. Now, for the contraction of $\mathscr{G}^{*}$, using $\left(A_{1}\right)$ and $\left(A_{3}\right)$ for any $y, z \in \mathcal{S}$, we have

$$
\begin{aligned}
& \left|\mathscr{G}^{*}(y)-\mathscr{G}^{*}(z)\right| \\
& \leq|g(y)-g(z)|+|h(y)-h(z)| \\
& \quad+\sum_{j=1}^{k}\left|(t-1) t_{j}\left[\widehat{I}_{j}\left(y\left(t_{j}\right)\right)-\widehat{I}_{j} z\left(t_{j}\right)\right]\right| \\
& \quad+\left|\sum_{j=1}^{k}(1-t)\left[I_{j}\left(y\left(t_{j}\right)\right)-I_{j} z\left(t_{j}\right)\right]\right| \\
& \leq K_{g}\|y-z\|+K_{h}\|y-z\|+k(\mathbb{A}+\mathbb{B})\|y-z\| \\
& \leq\left(K_{g}+K_{h}+k(\mathbb{A}+\mathbb{B})\right)\|y-z\| .
\end{aligned}
$$

Thus, $\mathscr{G}^{*}$ will be contraction if the following assumption holds:

$$
K_{g}+K_{h}+k(\mathbb{A}+\mathbb{B})<1 .
$$

Next $\mathscr{F}^{*}$ is compact. The continuity of $f$ implies that $\mathscr{F}^{*}$ is continuous. For $y, u \in \mathcal{S}$

$$
\begin{aligned}
& \left|\mathscr{F}^{*} y(t)\right| \leq \mid \frac{1}{\Gamma(\rho)} \int_{t_{j}}^{t}(t-\kappa)^{\rho-1} u(\kappa) d \kappa \\
& \quad+\frac{1}{\Gamma(\rho)} \sum_{j=1}^{k} \int_{t_{j-1}}^{t_{j}}\left(t_{j}-\kappa\right)^{\rho-1} u(\kappa) d \kappa \mid \\
& +\mid \frac{1}{\Gamma(\rho-1)} \sum_{j=1}^{k}\left(t-t_{j}\right) \int_{t_{j-1}}^{t_{j}}\left(t_{j}-\kappa\right)^{\rho-2} u(\kappa) d \kappa \\
& \quad-\frac{t}{\Gamma(\rho)} \sum_{j=1}^{k+1} \int_{t_{j-1}}^{t_{j}}\left(t_{j}-\kappa\right)^{\rho-1} u(\kappa) d \kappa \mid
\end{aligned}
$$

$$
\begin{aligned}
& +\left|\frac{t}{\Gamma(\rho-1)} \sum_{j=1}^{k}\left(1-t_{j}\right) \int_{t_{j-1}}^{t_{j}}\left(t_{j}-\kappa\right)^{\rho-2} u(\kappa) d \kappa\right| \\
& \leq \mid \frac{1}{\Gamma(\rho)} \int_{t_{j}}^{t}(t-\kappa)^{\rho-1} u(\kappa) d \kappa \\
& +\frac{1}{\Gamma(\rho)} \sum_{j=1}^{k} \int_{t_{j-1}}^{t_{j}}\left(t_{j}-\kappa\right)^{\rho-1} u(\kappa) d \kappa \mid \\
& +\left|\frac{1}{\Gamma(\rho-1)} \sum_{j=1}^{k}\left(t-t_{j}\right) \int_{t_{j-1}}^{t_{j}}\left(t_{j}-\kappa\right)^{\rho-2} u(\kappa) d \kappa\right| \\
& +\left|\frac{t}{\Gamma(\rho-1)} \sum_{j=1}^{k}\left(1-t_{j}\right) \int_{t_{j-1}}^{t_{j}}\left(t_{j}-\kappa\right)^{\rho-2} u(\kappa) d \kappa\right| .
\end{aligned}
$$

By using (55), we get

$$
\left\|\mathscr{F}^{*} y\right\| \leq \frac{(3 k+1) \mathcal{N}_{0}}{\Gamma(\rho)},
$$

which indicates that $\mathscr{F}^{*}$ is uniformly bounded on $\mathcal{S}$. Let us take a bounded subset $\mathcal{U}$ of $\mathcal{S}$ and $y \in \mathcal{U}$. Then for $t, t_{0} \in \mathfrak{J}$ with $0 \leq t \leq t_{0} \leq 1$, we have

$$
\begin{aligned}
& \left|\mathscr{F}^{*} y(t)-\mathscr{F}^{*} y\left(t_{0}\right)\right|=\mid \frac{1}{\Gamma(\rho)} \int_{t_{j}}^{t}(t-\kappa)^{\rho-1} u(\kappa) d \kappa \\
& -\frac{1}{\Gamma(\rho)} \int_{t_{j}}^{t_{0}}\left(t_{0}-\kappa\right)^{\rho-1} u(\kappa) d \kappa \\
& +\frac{1}{\Gamma(\rho-1)} \sum_{j=1}^{k}\left(t-t_{0}\right) \int_{t j-1}^{t_{j}}\left(t_{j}-\kappa\right)^{\rho-2} u(\kappa) d \kappa \\
& +\frac{\left(t-t_{0}\right)}{\Gamma(\rho-1)} \sum_{j=1}^{k}\left(1-t_{j}\right) \int_{t_{j-1}}^{t_{j}}\left(t_{j}-\kappa\right)^{\rho-2} u(\kappa) d \kappa \\
& +\frac{\left(t-t_{0}\right)}{\Gamma(\rho)} \sum_{j=1}^{k+1} \int_{t_{j-1}}^{t_{j}}\left(t_{j}-\kappa\right)^{\rho-1} u(\kappa) d \kappa \\
& \leq \frac{\mathcal{N}_{0}}{\Gamma(\rho+1)}\left|\int_{t_{j}}^{t}(t-\kappa)^{\rho-1} d \kappa-\int_{t_{j}}^{t_{0}}\left(t_{0}-\kappa\right)^{\rho-1} d \kappa\right| \\
& +\frac{\mathcal{N}_{0}\left(t-t_{0}\right)}{\Gamma(\rho-1)} \sum_{j=1}^{k}\left|\int_{t j-1}^{t_{j}}\left(t_{j}-\kappa\right)^{\rho-1} d \kappa\right| \\
& +\frac{\mathcal{N}_{0}\left(t-t_{0}\right)}{\Gamma(\rho-1)} \sum_{j=1}^{k}\left|\left(1-t_{j}\right) \int_{t_{j-1}}^{t_{j}}\left(t_{j}-\kappa\right)^{\rho-2} d \kappa\right| \\
& +\frac{\mathcal{N}_{0}\left(t-t_{0}\right)}{\Gamma(\rho)} \sum_{j=1}^{k+1}\left|\int_{t_{j-1}}^{t_{j}}\left(t_{j}-\kappa\right)^{\rho-1} d \kappa\right|,
\end{aligned}
$$


where

$$
\|u\| \leq \frac{n^{*}+o^{*} \xi^{*}}{1-p^{*}}=\mathcal{N}_{0} .
$$

Therefore, the right hand side is

$$
\left\|\mathscr{F}^{*}(y(t))-\mathscr{F}^{*}\left(y\left(t_{0}\right)\right)\right\| \longrightarrow 0 \quad \text { as } t \longrightarrow t_{0} .
$$

Thus, $\mathscr{F}^{*}$ is equicontinuous and by Arzela-Ascoli's Theorem [49], $\mathscr{F}^{*}$ is compact. By using Theorem 6 , the considered problem (7) has at least one positive solution.

\section{Ulam Stability Results}

In this section, we built up some sufficient conditions under which problem (7) satisfies the assumptions of various kinds of Ulam-Hyers stability.

Lemma 18. If $z \in \mathbb{X}$ is the solution of inequality (17) and $1<$ $\rho \leq 2$, then $z$ is the solution of the following inequality:

$$
|z(t)-\nu(t)| \leq\left(\frac{4 k+2 k \Gamma(\rho)+2}{\Gamma(\rho)}\right) \epsilon .
$$

Proof. Since $z$ is the solution of inequality (17), so in view of Remark 13, we have

$$
\begin{aligned}
{ }^{c} D^{\rho} z(t) & =f\left(t, z(t),{ }^{c} D^{\rho} z(t)\right)+\vartheta(t), \\
t \in \mathfrak{I}, t \neq t_{j}, \quad j=1,2, \ldots, k, & \\
\Delta z\left(t_{j}\right) & =I_{j}\left(z\left(t_{j}\right)\right)+\vartheta_{j}, \quad j=1,2, \ldots, k, \\
\Delta z^{\prime}\left(t_{j}\right) & =\widehat{I}_{j}\left(z\left(t_{j}\right)\right)+\vartheta_{j}, \quad j=1,2, \ldots, k, \\
z(0) & =g(z), \\
z(1) & =h(z) .
\end{aligned}
$$

So the solution of (65) will be in the following form:

$$
\begin{aligned}
= & \frac{1}{\Gamma(\rho)} \int_{t_{j}}^{t}(t-\kappa)^{\rho-1} u(\kappa) d \kappa \\
& +\frac{1}{\Gamma(\rho)} \int_{t_{j}}^{t}(t-\kappa)^{\rho-1} \vartheta(\kappa) d \kappa \\
& +\frac{1}{\Gamma(\rho)} \sum_{j=1}^{k} \int_{t_{j-1}}^{t_{j}}\left(t_{j}-\kappa\right)^{\rho-1} u(\kappa) d \kappa \\
& +\frac{1}{\Gamma(\rho)} \sum_{j=1}^{k} \int_{t_{j-1}}^{t_{j}}\left(t_{j}-\kappa\right)^{\rho-1} \vartheta(\kappa) d \kappa \\
& +\frac{1}{\Gamma(\rho-1)} \sum_{j=1}^{k}\left(t-t_{j}\right) \int_{t_{j-1}}^{t_{j}}\left(t_{j}-\kappa\right)^{\rho-2} u(\kappa) d \kappa \\
& +\frac{1}{\Gamma(\rho-1)} \sum_{j=1}^{k}\left(t-t_{j}\right) \int_{t_{j-1}}^{t_{j}}\left(t_{j}-\kappa\right)^{\rho-2} \vartheta(\kappa) d \kappa
\end{aligned}
$$

$$
\begin{aligned}
& -\frac{t}{\Gamma(\rho)} \sum_{j=1}^{k+1} \int_{t_{j-1}}^{t_{j}}\left(t_{j}-\kappa\right)^{\rho-1} u(\kappa) d \kappa \\
& -\frac{t}{\Gamma(\rho)} \sum_{j=1}^{k+1} \int_{t_{j-1}}^{t_{j}}\left(t_{j}-\kappa\right)^{\rho-1} \vartheta(\kappa) d \kappa \\
& -\frac{t}{\Gamma(\rho-1)} \sum_{j=1}^{k}\left(1-t_{j}\right) \int_{t_{j-1}}^{t_{j}}\left(t_{j}-\kappa\right)^{\rho-2} u(\kappa) d \kappa \\
& -\frac{t}{\Gamma(\rho-1)} \sum_{j=1}^{k}\left(1-t_{j}\right) \int_{t_{j-1}}^{t_{j}}\left(t_{j}-\kappa\right)^{\rho-2} \vartheta(\kappa) d \kappa \\
& +t g(z)+(1-t) h(z)+\sum_{j=1}^{k}\left(t-t_{j}\right) \widehat{I}_{j}\left(z\left(t_{j}\right)\right) \\
& +\sum_{j=1}^{k}\left(t-t_{j}\right) \vartheta_{j}-\sum_{j=1}^{k} t\left(1-t_{j}\right) \widehat{I}_{j} z\left(t_{j}\right) \\
& -\sum_{j=1}^{k} t I_{j} z\left(t_{j}\right)-\sum_{j=1}^{k} t \vartheta_{j}, \\
& +\sum_{j=1}^{k} t\left(1-t_{j}\right) \vartheta_{j}+\sum_{j=1}^{k} I_{j}\left(z\left(t_{j}\right)\right)+\sum_{j=1}^{k} \vartheta_{j} \\
& +k
\end{aligned}
$$

where

$$
u(t)=f(t, z(t), u(t))
$$

For convenience, we denote the sum of terms free of $\vartheta$ by $\nu(t)$, that is,

$$
\begin{aligned}
v(t) & \frac{1}{\Gamma(\rho)} \int_{t_{j}}^{t}(t-\kappa)^{\rho-1} u(\kappa) d \kappa \\
& +\frac{1}{\Gamma(\rho)} \sum_{j=1}^{k} \int_{t_{j-1}}^{t_{j}}\left(t_{j}-\kappa\right)^{\rho-1} u(\kappa) d \kappa \\
& +\frac{1}{\Gamma(\rho-1)} \sum_{j=1}^{k}\left(t-t_{j}\right) \int_{t_{j-1}}^{t_{j}}\left(t_{j}-\kappa\right)^{\rho-2} u(\kappa) d \kappa \\
& +\frac{t}{\Gamma(\rho)} \sum_{j=1}^{k+1} \int_{t_{j-1}}^{t_{j}}\left(t_{j}-\kappa\right)^{\rho-1} u(\kappa) d \kappa \\
& +\frac{t}{\Gamma(\rho-1)} \sum_{j=1}^{k}\left(1-t_{j}\right) \int_{t_{j-1}}^{t_{j}}\left(t_{j}-\kappa\right)^{\rho-2} u(\kappa) d \kappa \\
& +\operatorname{tg}(z)+(1-t) h(z)+\sum_{j=1}^{k}\left(t-t_{j}\right) \widehat{I}_{j}\left(z\left(t_{j}\right)\right)
\end{aligned}
$$




$$
\begin{aligned}
& +\sum_{j=1}^{k} t\left(1-t_{j}\right) \widehat{I}_{j} z\left(t_{j}\right)+\sum_{j=1}^{k} I_{j}\left(z\left(t_{j}\right)\right) \\
& +\sum_{j=1}^{k} t I_{j} z\left(t_{j}\right) .
\end{aligned}
$$

Therefore, (66) becomes

$$
\begin{aligned}
& |z(t)-\nu(t)| \leq \mid \frac{1}{\Gamma(\rho)} \int_{t_{j}}^{t}(t-\kappa)^{\rho-1} \vartheta(\kappa) d \kappa \\
& +\frac{1}{\Gamma(\rho)} \sum_{j=1}^{k} \int_{t_{j-1}}^{t_{j}}\left(t_{j}-\kappa\right)^{\rho-1} \vartheta(\kappa) d \kappa \\
& +\frac{1}{\Gamma(\rho-1)} \sum_{j=1}^{k}\left(t-t_{j}\right) \int_{t_{j-1}}^{t_{j}}\left(t_{j}-\kappa\right)^{\rho-2} \vartheta(\kappa) d \kappa \\
& -\frac{t}{\Gamma(\rho)} \sum_{j=1}^{k+1} \int_{t_{j-1}}^{t_{j}}\left(t_{j}-\kappa\right)^{\rho-1} \vartheta(\kappa) d \kappa \\
& \quad-\frac{t}{\Gamma(\rho-1)} \sum_{j=1}^{k}\left(1-t_{j}\right) \int_{t_{j-1}}^{t_{j}}\left(t_{j}-\kappa\right)^{\rho-2} \vartheta(\kappa) d \kappa \\
& +\sum_{j=1}^{k}\left(t-t_{j}\right) \vartheta_{j}-\sum_{j=1}^{k} t\left(1-t_{j}\right) \vartheta_{j}+\sum_{j=1}^{k} \vartheta_{j}-\sum_{j=1}^{k} t \vartheta_{j} \mid
\end{aligned}
$$

By using (i) of Remark 13, we get

$$
|z(t)-v(t)| \leq\left(\frac{4 k+2 k \Gamma(\rho)+2}{\Gamma(\rho)}\right) \epsilon
$$

Theorem 19. Let assumptions $\left(A_{1}\right)-\left(A_{3}\right)$ hold along with the condition

$$
\frac{(4 k+2) L_{f}}{\left(1-K_{f}\right) \Gamma(\rho)}+K_{g}+K_{h}+k(\mathbb{A}+\mathbb{B}) \neq 1 .
$$

Then problem (7) will be Ulam-Hyers stable.

Proof. Suppose $z \in \mathbb{X}$ be any solution of inequality (17) and let $y$ be the unique solution of the considered problem (7), then

$$
\begin{aligned}
{ }^{c} D^{\rho} y(t) & =f\left(t, y(t),{ }^{c} D^{\rho} y(t)\right), \\
t \in \mathfrak{J}, t \neq t_{j}, j=1,2, \ldots, k, & \\
\Delta y\left(t_{j}\right) & =I_{j}\left(y\left(t_{j}\right)\right), \\
\Delta y^{\prime}\left(t_{j}\right) & =\widehat{I}_{j}\left(y\left(t_{j}\right)\right), \\
y(0) & =g(y), \\
y(1) & =h(y) .
\end{aligned}
$$

Now

$$
\begin{aligned}
|z(t)-y(t)| & =|z(t)-v(t)+v(t)-y(t)| \\
& \leq|z(t)-v(t)|+|v(t)-y(t)| .
\end{aligned}
$$

Using Lemma 18 in (73), we have

$$
\begin{aligned}
& |z(t)-y(t)| \leq\left(\frac{4 k+2 k \Gamma(\rho)+2}{\Gamma(\rho)}\right) \epsilon+\frac{1}{\Gamma(\rho)} \\
& \quad \cdot \int_{t_{j}}^{t}(t-\kappa)^{\rho-1}|u(\kappa)-q(\kappa)| d \kappa+\frac{1}{\Gamma(\rho)} \\
& \quad \cdot \sum_{j=1}^{k} \int_{t_{j-1}}^{t_{j}}\left(t_{j}-\kappa\right)^{\rho-1}|u(\kappa)-q(\kappa)| d \kappa+\frac{1}{\Gamma(\rho-1)} \\
& \quad \cdot \sum_{j=1}^{k}\left(t-t_{j}\right) \int_{t_{j-1}}^{t_{j}}\left(t_{j}-\kappa\right)^{\rho-2}|u(\kappa)-q(\kappa)| d \kappa \\
& +\frac{t}{\Gamma(\rho)} \sum_{j=1}^{k+1} \int_{t_{j-1}}^{t_{j}}\left(t_{j}-\kappa\right)^{\rho-1}|u(\kappa)-q(\kappa)| d \kappa \\
& +\sum_{j=1}^{k}|t|\left|I_{j}\left(z\left(t_{j}\right)\right)-I_{j}\left(y\left(t_{j}\right)\right)\right| \\
& \quad+\sum_{j=1}^{k}\left|t\left(1-t_{j}\right)\right|\left|I_{j}\left(z\left(t_{j}\right)\right)-\widehat{I}_{j}\left(y\left(t_{j}\right)\right)\right| \\
& +\int_{t_{j-1}}^{t_{j}}\left(t_{j}-\kappa\right)^{\rho-2}|u(\kappa)-q(\kappa)| d \kappa+|t| \\
& +|g(z)-g(y)|+|(1-t)||h(z)-h(y)| \\
& +\sum_{j=1}^{k}\left|\left(t-t_{j}\right)\right|\left|\widehat{I}_{j}\left(z\left(t_{j}\right)\right)-\widehat{I}_{j}\left(y\left(t_{j}\right)\right)\right|
\end{aligned}
$$

for $u, q \in \mathbb{X}$, where

$$
\begin{aligned}
& u(t)=f(t, z(t), u(t)), \\
& q(t)=f(t, y(t), q(t)) .
\end{aligned}
$$

Now by $\left(A_{2}\right)$, we have

$$
\begin{aligned}
|u(t)-q(t)| & =|f(t, z(t), u(t))-f(t, y(t), q(t))| \\
& \leq L_{f}|z(t)-y(t)|+K_{f}|u(t)-q(t)| .
\end{aligned}
$$

So, we obtain

$$
|u(t)-q(t)| \leq \frac{K_{f}}{1-L_{f}}|z(t)-y(t)| .
$$


Using $\left(A_{1}\right),\left(A_{3}\right)$ and (77) in (74), so for every $t \in \mathfrak{J}$, we get the following:

$$
\begin{aligned}
\|z-y\| \leq & \left(\frac{4 k+2 k \Gamma(\rho)+2}{\Gamma(\rho)}\right) \epsilon \\
& +\frac{L_{f}}{\left(1-K_{f}\right) \Gamma(\rho+1)}\|z-y\| \\
& +\frac{k L_{f}}{\left(1-K_{f}\right) \Gamma(\rho+1)}\|z-y\|
\end{aligned}
$$

$$
\begin{aligned}
& +\frac{k L_{f}}{\left(1-K_{f}\right) \Gamma(\rho)}\|z-y\| \\
& +\frac{(k+1) L_{f}}{\left(1-K_{f}\right) \Gamma(\rho+1)}\|z-y\| \\
& +\frac{k L_{f}}{\left(1-K_{f}\right) \Gamma(\rho)}\|z-y\|+K_{g}\|z-y\| \\
& +K_{h}\|z-y\|+k(\mathbb{A}+\mathbb{B})\|z-y\| .
\end{aligned}
$$

After simplification, we obtain

$$
\|z-y\| \leq\left[\frac{(4 k+2 k \Gamma(\rho)+2) / \Gamma(\rho)}{1-\left((4 k+2) L_{f} /\left(1-K_{f}\right) \Gamma(\rho)+K_{g}+K_{h}+k(\mathbb{A}+\mathbb{B})\right)}\right] \epsilon .
$$

Hence, we have

$$
|z(t)-y(t)| \leq c_{f, k} \epsilon,
$$

where

$$
\begin{aligned}
& c_{f, k} \\
& =\frac{(4 k+2 k \Gamma(\rho)+2) / \Gamma(\rho)}{1-\left((4 k+2) L_{f} /\left(1-K_{f}\right) \Gamma(\rho)+K_{g}+K_{h}+k(\mathbb{A}+\mathbb{B})\right)},
\end{aligned}
$$

with

$$
\frac{(4 k+2) L_{f}}{\left(1-K_{f}\right) \Gamma(\rho)}+K_{g}+K_{h}+k(\mathbb{A}+\mathbb{B}) \neq 1 .
$$

Thus, problem (7) is Ulam-Hyers stable.

$$
\begin{aligned}
& +\frac{1}{\Gamma(\rho-1)} \sum_{j=1}^{k}\left(t-t_{j}\right) \int_{t_{j-1}}^{t_{j}}\left(t_{j}-\kappa\right)^{\rho-2} \vartheta(\kappa) d \kappa \\
& -\frac{t}{\Gamma(\rho)} \sum_{j=1}^{k+1} \int_{t_{j-1}}^{t_{j}}\left(t_{j}-\kappa\right)^{\rho-1} \vartheta(\kappa) d \kappa \\
& -\frac{t}{\Gamma(\rho-1)} \sum_{j=1}^{k}\left(1-t_{j}\right) \int_{t_{j-1}}^{t_{j}}\left(t_{j}-\kappa\right)^{\rho-2} \vartheta(\kappa) d \kappa \\
& +\sum_{j=1}^{k}\left(t-t_{j}\right) \vartheta_{j}-\sum_{j=1}^{k} t\left(1-t_{j}\right) \vartheta_{j}+\sum_{j=1}^{k} \vartheta_{j}-\sum_{j=1}^{k} t \vartheta_{j} \mid .
\end{aligned}
$$

Remark 20. By setting $\phi_{f, k}(\epsilon)=c_{f, k} \epsilon, \phi_{f, k}(0)=0$ in (80), then by Definition 9 the considered problem (7) is generalized Ulam-Hyers stable.

$\left(A_{8}\right)$ Suppose a function $\varphi \in\left(\mathfrak{I}, \mathbb{R}^{+}\right)$, which is increasing. Then there is $\eta_{\varphi}>0$, such that, for every $t \in \mathfrak{I}$, the following integral inequality

$$
I^{\rho} \varphi(t) \leq \eta_{\varphi} \varphi(t) \text { accordingly } I^{\rho-1} \varphi(t) \leq \eta_{\varphi} \varphi(t)
$$

holds.

Lemma 21. Let assumption $\left(A_{8}\right)$ hold and suppose $z \in \mathbb{X}$ is the solution of inequality (18), then $z$ will be the solution of the following integral inequality:

$$
|z(t)-\nu(t)| \leq\left((4 k+2) \eta_{\varphi}+2 k \wp\right) \varphi(t) \epsilon .
$$

Proof. From Lemma 18, we have

$$
\begin{gathered}
|z(t)-\nu(t)| \leq \mid \frac{1}{\Gamma(\rho)} \int_{t_{j}}^{t}(t-\kappa)^{\rho-1} \vartheta(\kappa) d \kappa \\
+\frac{1}{\Gamma(\rho)} \sum_{j=1}^{k} \int_{t_{j-1}}^{t_{j}}\left(t_{j}-\kappa\right)^{\rho-1} \vartheta(\kappa) d \kappa
\end{gathered}
$$

By Remark 14 and $\left(A_{8}\right)$, we obtain

$$
\begin{aligned}
|z(t)-\nu(t)| \leq & \eta_{\varphi} \varphi(t) \epsilon+k \eta_{\varphi} \varphi(t) \epsilon+k \eta_{\varphi} \varphi(t) \epsilon \\
& +(k+1) \eta_{\varphi} \varphi(t) \epsilon+k \eta_{\varphi} \varphi(t) \epsilon \\
& +2 k \wp \epsilon .
\end{aligned}
$$

Hence, we have

$$
|z(t)-v(t)| \leq\left((4 k+2) \eta_{\varphi}+2 k_{\wp}\right) \varphi(t) \epsilon .
$$

Theorem 22. Suppose assumptions $\left(A_{1}\right)-\left(A_{3}\right),\left(A_{5}\right)$, and $\left(A_{8}\right)$ with

$$
\frac{(4 k+2) L_{f}}{\left(1-K_{f}\right) \Gamma(\rho)}+K_{g}+K_{h}+k(\mathbb{A}+\mathbb{B}) \neq 1
$$

hold. Then the considered problem (7) will be Ulam-HyersRassias stable. 
Proof. Suppose $z \in \mathbb{X}$ be any solution of inequality (17) and $y$ be the unique solution of the considered problem (7), then from (73)

$$
|z(t)-y(t)| \leq|z(t)-v(t)|+|\nu(t)-y(t)|
$$

Now using Lemma 21 in (89), we obtain

$$
\begin{aligned}
& |z(t)-y(t)| \leq\left((4 k+2) \eta_{\varphi}+2 k \wp\right) \varphi(t) \epsilon+\frac{1}{\Gamma(\rho)} \\
& \cdot \int_{t_{j}}^{t}(t-\kappa)^{\rho-1}|u(\kappa)-q(\kappa)| d \kappa+\frac{1}{\Gamma(\rho)} \\
& \cdot \sum_{j=1}^{k} \int_{t_{j-1}}^{t_{j}}\left(t_{j}-\kappa\right)^{\rho-1}|u(\kappa)-q(\kappa)| d \kappa+\frac{1}{\Gamma(\rho-1)} \\
& \cdot \sum_{j=1}^{k}\left(t-t_{j}\right) \int_{t_{j-1}}^{t_{j}}\left(t_{j}-\kappa\right)^{\rho-2}|u(\kappa)-q(\kappa)| d \kappa \\
& +\frac{t}{\Gamma(\rho)} \sum_{j=1}^{k+1} \int_{t_{j-1}}^{t_{j}}\left(t_{j}-\kappa\right)^{\rho-1}|u(\kappa)-q(\kappa)| d \kappa \\
& +\frac{t}{\Gamma(\rho-1)} \sum_{j=1}^{k}\left(1-t_{j}\right) \\
& \cdot \int_{t_{j-1}}^{t_{j}}\left(t_{j}-\kappa\right)^{\rho-2}|u(\kappa)-q(\kappa)| d \kappa+|t| \\
& \cdot|g(z)-g(y)|+|(1-t)||h(z)-h(y)| \\
& +\sum_{j=1}^{k}\left|\left(t-t_{j}\right)\right|\left|\widehat{I}_{j}\left(z\left(t_{j}\right)\right)-\widehat{I}_{j}\left(y\left(t_{j}\right)\right)\right| \\
& +\sum_{j=1}^{k}\left|t\left(1-t_{j}\right)\right|\left|\widehat{I}_{j}\left(z\left(t_{j}\right)\right)-\widehat{I}_{j}\left(y\left(t_{j}\right)\right)\right|
\end{aligned}
$$

$$
\begin{aligned}
& +\sum_{j=1}^{k}\left|I_{j}\left(z\left(t_{j}\right)\right)-I_{j}\left(y\left(t_{j}\right)\right)\right| \\
& +\sum_{j=1}^{k}|t|\left|I_{j}\left(z\left(t_{j}\right)\right)-I_{j}\left(y\left(t_{j}\right)\right)\right|,
\end{aligned}
$$

for $u, q \in \mathbb{X}$, where

$$
\begin{aligned}
& u(t)=f(t, z(t), u(t)), \\
& q(t)=f(t, y(t), q(t)) .
\end{aligned}
$$

Using $\left(A_{1}\right)-\left(A_{3}\right)$ and $\left(A_{5}\right)$ likewise in Theorem 19 , so (90) becomes

$$
\begin{aligned}
\|z-y\| \leq & \left((4 k+2) \eta_{\varphi}+2 k \wp\right) \varphi(t) \epsilon \\
& +\frac{L_{f}}{\left(1-K_{f}\right) \Gamma(\rho+1)}\|z-y\| \\
& +\frac{k L_{f}}{\left(1-K_{f}\right) \Gamma(\rho+1)}\|z-y\| \\
& +\frac{k L_{f}}{\left(1-K_{f}\right) \Gamma(\rho)}\|z-y\| \\
& +\frac{(k+1) L_{f}}{\left(1-K_{f}\right) \Gamma(\rho+1)}\|z-y\| \\
& +\frac{k L_{f}}{\left(1-K_{f}\right) \Gamma(\rho)}\|z-y\|+K_{g}\|z-y\| \\
& +K_{h}\|z-y\|+k(\mathbb{A}+\mathbb{B})\|z-y\| .
\end{aligned}
$$

After arrangement, we obtain the following:

$$
\|z-y\| \leq\left[\frac{(4 k+2) \eta_{\varphi}+2 k_{\wp}}{1-\left((4 k+2) L_{f} /\left(1-K_{f}\right) \Gamma(\rho)+K_{g}+K_{h}+k(\mathbb{A}+\mathbb{B})\right)}\right] \varphi(t) \epsilon .
$$

Hence, we have

$$
|z(t)-y(t)| \leq c_{f, k, \varphi} \varphi(t) \epsilon,
$$

where

$$
\begin{aligned}
& c_{f, k, \varphi} \\
& =\frac{(4 k+2) \eta_{\varphi}+2 k_{\wp}}{1-\left((4 k+2) L_{f} /\left(1-K_{f}\right) \Gamma(\rho)+K_{g}+K_{h}+k(\mathbb{A}+\mathbb{B})\right)}, \\
& \frac{(4 k+2) L_{f}}{\left(1-K_{f}\right) \Gamma(\rho)}+K_{g}+K_{h}+k(\mathbb{A}+\mathbb{B}) \neq 1 .
\end{aligned}
$$

Hence, the considered problem (7) is Ulam-Hyers-Rassias stable.

Remark 23. If we plug $\epsilon=1$ in (94), then by Definition 11 the considered problem (7) will be generalized Ulam-Hyers-Rassias stable.

\section{Example}

Consider the following implicit impulsive fractional differential equations with nonlocal boundary conditions. 
Example 1.

$$
\begin{aligned}
{ }^{c} D^{4 / 3} y(t)= & \frac{|y(t)|}{30(t+2)(1+|y(t)|)} \\
& +\frac{\cos \left|{ }^{c} D^{4 / 3} y(t)\right|}{30+t^{2}}, \quad t \in[0,1], t \neq \frac{1}{2}, \\
y(0)= & g(y)=\sum_{j=1}^{k} a_{j} y\left(\xi_{j}\right), \\
u(1)= & h(u)=\sum_{j=1}^{k} b_{j} y\left(\eta_{j}\right), \\
\Delta y\left(\frac{1}{2}\right)= & I y\left(\frac{1}{2}\right)=\frac{|y(1 / 2)|}{60+|y(1 / 2)|}, \\
\Delta y^{\prime}\left(\frac{1}{2}\right)= & \widehat{I} y\left(\frac{1}{2}\right)=\frac{|y(1 / 2)|}{30+|y(1 / 2)|},
\end{aligned}
$$

where $\sum_{j=1}^{k} a_{j}<10^{-1}, \sum_{j=1}^{k} b_{j}<10^{-1}$, and $\xi_{j}, \eta_{j} \in(0,1)$, $\xi, \eta \neq 1 / 2$.

For $y, z \in X$, we have

$$
\begin{aligned}
& |g(y)-g(z)| \leq \frac{1}{10}\|y-z\|, \\
& |h(y)-h(z)| \leq \frac{1}{10}\|y-z\| .
\end{aligned}
$$

Hence, $\left(A_{1}\right)$ is satisfied with $K_{g}=1 / 10$ and $K_{h}=1 / 10$.

Now for any $y, \bar{y}, z, \bar{z} \in \mathbb{R}, t \in \mathfrak{I}$, we get

$$
|f(t, y, z)-f(t, \bar{y}, \bar{z})| \leq \frac{1}{30}\|y-\bar{y}\|+\frac{1}{30}\|z-\bar{z}\|,
$$

so $\left(A_{2}\right)$ is satisfied with $L_{f}=K_{f}=1 / 30$.

Also, for $y, z \in \mathbb{R}$, we have

$$
\begin{aligned}
& |I(y)-I(z)| \leq \frac{1}{60}\|y-z\|, \\
& |\widehat{I}(y)-\widehat{I}(z)| \leq \frac{1}{30}\|y-z\| .
\end{aligned}
$$

Hence, $\left(A_{3}\right)$ is also satisfied with $\mathbb{A}=1 / 60$ and $\mathbb{B}=1 / 30$.

Therefore, we can see for $K_{g}=1 / 10, K_{h}=1 / 10, \mathbb{A}=$ $1 / 60, \mathbb{B}=1 / 30, k=1, \rho=4 / 3$, and $L_{f}=K_{f}=1 / 30$

$$
\begin{aligned}
& \frac{(4 k+2) L_{f}}{\left(1-K_{f}\right) \Gamma(\rho)}+k(\mathbb{A}+\mathbb{B})+K_{g}+K_{h} \approx 0.44308 \\
& \quad<1 .
\end{aligned}
$$

Thus, condition (39) is satisfied. So problem (96) has a unique positive solution.

Furthermore, $c_{f, k}>0$ with condition (17) holds and

$$
\frac{(4 k+2) L_{f}}{\left(1-K_{f}\right) \Gamma(\rho)}+k(\mathbb{A}+\mathbb{B})+K_{g}+K_{h} \approx 0.44308
$$

$$
\neq 1 \text {. }
$$

So by Theorem 19 problem (96) is Ulam-Hyers stable and by Remark 20, it will be generalized Ulam-Hyers stable. Also by demonstrating the conditions of Theorem 22 and Remark 23, it can be easily seen that the considered problem (96) is Ulam-Hyers-Rassias stable and generalized Ulam-Hyers-Rassias stable.

\section{Conclusion}

We have successfully built up some proper conditions for existence theory of implicit impulsive fractional order differential equations with nonlocal boundary conditions, by using different kinds of fixed point theorems which are stated in Section 2. The concerned theorems ensure the existence and uniqueness of solution. Further, we did settle some adequate conditions for different kinds of Ulam-Hyers stability (see Theorems 19 and 22 with Remarks 20 and 23 , respectively) by using Definitions $8-11$. The mentioned stability is rarely investigated for implicit impulsive fractional differential equations and also very important. Finally, we illustrated the main results by giving a suitable example.

\section{Conflicts of Interest}

There are no conflicts of interest regarding this research work.

\section{Authors' Contributions}

All authors contributed equally to this manuscript and have read and approved the final version.

\section{Acknowledgments}

This research work has been supported by the Institution of Business Administration, University Road, Karachi 74200, Pakistan. The last author Dr. Ghaus ur Rahman is supported by HEC via Project no. 21-190/SRGP/R\&D/HEC/2014.

\section{References}

[1] R. Lewandowski and B. Chorążyczewski, "Identification of the parameters of the Kelvin-Voigt and the Maxwell fractional models, used to modeling of viscoelastic dampers," Computers and Structures, vol. 88, no. 1-2, pp. 1-17, 2010.

[2] F. Yu, "Integrable coupling system of fractional soliton equation hierarchy," Physics Letters A, vol. 373, no. 41, pp. 3730-3733, 2009.

[3] R. Hilfer, Applications of Fractional Calculus in Physics, World Scientific, Singapore, 2000.

[4] A. A. Kilbas, H. M. Srivastava, and J. J. Trujillo, Theory and Applications of Fractional Differential Equations, vol. 204 of North-Holland athematics Studies, Elsevier, New York, NY, USA, 2006.

[5] V. Lakshmikantham, S. Leela, and J. Vasundhara, Theory of Fractional Dynamic Systems, Cambridge Academic Publishers, Cambridge, UK, 2009.

[6] K. S. Miller and B. Ross, An Introduction to the Fractional Calculus and Fractional Differential Equations, Wiley, New York, NY, USA, 1993. 
[7] I. Podlubny, Fractional Differential Equations, Mathematics in Science and Engineering, Academic Press, New York, NY, USA, 1999.

[8] A. M. El-Sayed and E. O. Bin-Taher, "Positive solutions for a nonlocal multi-point boundary-value problem of fractional and second order," Electronic Journal of Differential Equations, vol. 64, pp. 1-8, 2013.

[9] R. A. Khan and K. Shah, "Existence and uniqueness of solutions to fractional order multi-point boundary value problems," Communications in Applied Analysis, vol. 19, pp. 515-526, 2015.

[10] M. Rehman and R. Khan, "A note on boundary value problems for a coupled system of fractional differential equations," International Journal of Applied Mathematics and Computer Science, vol. 61, no. 9, pp. 2630-2637, 2011.

[11] K. Shah, N. Ali, and R. A. Khan, "Existence of positive solution to a class of fractional differential equations with three point boundary conditions," Applied Mathematics Letters, vol. 5, no. 3, pp. 291-296, 2016.

[12] K. Shah and R. A. Khan, "Existence and uniqueness of positive solutions to a coupled system of nonlinear fractional order differential equations with anti periodic boundary conditions," Journal of Difference Equations and Applications, vol. 7, no. 2, pp. 245-262, 2015.

[13] K. Shah, H. Khalil, and R. A. Khan, "Upper and lower solutions to a coupled system of nonlinear fractional differential equations," Progress in Fractional Differentiation and Applications, vol. 1, no. 1, pp. 1-8, 2016.

[14] X. Su, "Boundary value problem for a coupled system of nonlinear fractional differential equations," Applied Mathematics Letters, vol. 22, no. 1, pp. 64-69, 2009.

[15] H. Akça, R. Alassar, V. Covachev, Z. Covacheva, and E. Al-Zahrani, "Continuous-time additive Hopfield-type neural networks with impulses," Journal of Mathematical Analysis and Applications, vol. 290, no. 2, pp. 436-451, 2004.

[16] A. Andronov, A. Witt, and S. Haykin, Oscilation Theory, Nauka, Moscow, Russia, 1981.

[17] D. D. Bainov, M. B. Dimitrova, and A. B. Dishliev, "Oscillation of the bounded solutions of impulsive differential-difference equations of second order," Applied Mathematics and Computation, vol. 114, no. 1, pp. 61-68, 2000.

[18] V. Babitskii and V. Krupenin, Vibration in Strongly Nonlinear Systems, Nauka, Moskow, Russia, 1985.

[19] F. Chernousko, L. Akulenko, and B. Sokolov, Control of Oscillations, Nauka, Moskow, Russia, 1980.

[20] L. O. Chua and L. Yang, "Cellular Neural Networks: Applications," IEEE Transactions on Circuits and Systems II: Express Briefs, vol. 35, no. 10, pp. 1273-1290, 1988.

[21] V. Lakshmikantham, D. D. Bainov, and P. S. Simeonov, Theory of Impulsive Differential Equations, World Scientific, Singapore, 1989.

[22] E. Popov, The Dynamics of Automatic Control Systems, Gostehizdat, Moskow, Russia, 1964.

[23] S. Zavalishchin and A. Sesekin, Impulsive processes: Models and applications, Nauka, Moskow, Russia, 1991.

[24] N. I. Yurchuk, "Solvability of boundary value problems for certain operatordifferential equations," Differentsial'nye uravneniya, vol. 13, no. 4, pp. 626-636, 1977.

[25] K. Diethelm and N. J. Ford, "Analysis of fractional differential equations," Journal of Mathematical Analysis and Applications, vol. 265, no. 2, pp. 229-248, 2002.
[26] S. Momani and R. W. Ibrahim, "On a fractional integral equation of periodic functions involving Weyl-Riesz operator in Banach algebras," Journal of Mathematical Analysis and Applications, vol. 339, no. 2, pp. 1210-1219, 2008.

[27] R. W. Ibrahim and S. Momani, "On the existence and uniqueness of solutions of a class of fractional differential equations," Journal of Mathematical Analysis and Applications, vol. 334, no. 1, pp. 1-10, 2007.

[28] B. Bonilla, M. Rivero, and J. J. Trujillo, "On systems of linear fractional differential equations with constant coefficients," Applied Mathematics and Computation, vol. 187, no. 1, pp. 6878, 2007.

[29] Y. Tian and Z. Bai, "Impulsive boundary value problem for differential equations with fractional order," Differential Equations and Dynamical Systems, vol. 21, no. 3, pp. 253-260, 2013.

[30] S. M. Ulam, A Collection of Mathematical Problems, Interscience, New York, NY, USA, 1960.

[31] D. H. Hyers, "On the stability of the linear functional equation," Proceedings of the National Acadamy of Sciences of the United States of America, vol. 27, pp. 222-224, 1941.

[32] D. H. Hyers, G. Isac, and T. M. Rassias, Stability of Functional Equations in Several Variables, Birkhäuser, Basel, Switzerland, 1998.

[33] J. Huang, S.-M. Jung, and Y. Li, "On Hyers-Ulam stability of nonlinear differential equations," Bulletin of the Korean Mathematical Society, vol. 52, no. 2, pp. 685-697, 2015.

[34] S.-M. Jung, Hyers-Ulam-Rassias Stability of Functional Equations in Mathematical Analysis, Hadronic Press, Palm Harbor, Florida, USA, 2001.

[35] S.-M. Jung, Hyers-Ulam-Rassias Stability of Functional Equations in Nonlinear Analysis, Springer, New York, NY, USA, 2011.

[36] T. Li and A. Zada, "Connections between Hyers-Ulam stability and uniform exponential stability of discrete evolution families of bounded linear operators over Banach spaces," Advances in Difference Equations, vol. 2016, no. 1, article no. 153, pp. 1-8, 2016.

[37] S. Tang, A. Zada, S. Faisal, M. M. El-Sheikh, and T. Li, "Stability of higher-order nonlinear impulsive differential equations," Journal of Nonlinear Science and its Applications, vol. 9, pp. 47134721, 2016.

[38] A. Zada, S. Faisal, and Y. Li, "On the Hyers-Ulam stability of first-order impulsive delay differential equations," Journal of Function Spaces, vol. 2016, Article ID 8164978, 6 pages, 2016.

[39] A. Zada, O. Shah, and R. Shah, "Hyers-Ulam stability of non-autonomous systems in terms of boundedness of Cauchy problems," Applied Mathematics and Computation, vol. 271, pp. 512-518, 2015.

[40] S. d. Abbas and M. Benchohra, "On the generalized UlamHyers-Rassias stability for Darboux problem for partial fractional implicit differential equations," Applied Mathematics ENotes, vol. 14, pp. 20-28, 2014.

[41] Z. Ali, A. Zada, and K. Shah, "Existence and Stability Analysis of Three Point Boundary Value PROblem," International Journal of Applied and Computational Mathematics, vol. 3, no. suppl. 1, pp. 651-664, 2017.

[42] M. Benchohra and S. Bouriah, "Existence and stability results for nonlinear boundary value problem for implicit differential equations of fractional order," Moroccan Journal of Pure and Applied Analysis, vol. 1, no. 1, pp. 22-37, 2015.

[43] R. W. Ibrahim, "Generalized Ulam-Hyers stability for fractional differential equations," International Journal of Mathematics, vol. 23, no. 5, 9 pages, 2012. 
[44] J. Wang, L. Lv, and Y. Zhou, "Ulam stability and data dependence for fractional differential equations with caputo derivative," Electronic Journal of Qualitative Theory of Differential Equations, vol. 63, pp. 1-10, 2011.

[45] J. Wang, Y. Zhou, and M. Feckan, "Nonlinear impulsive problems for fractional differential equations and Ulam stability," Computers and Mathematics with Applications, vol. 64, no. 10, pp. 3389-3405, 2012.

[46] Y. Li, Y. Chen, and I. Podlubny, "Stability of fractional-order nonlinear dynamic systems: Lyapunov direct method and generalized Mittag-LEFfler stability," Computers and Mathematics with Applications, vol. 59, no. 5, pp. 1810-1821, 2010.

[47] D. R. Smart, Fixed Point Theorems, Cambridge University Press, London, UK, 1980.

[48] I. A. Rus, "Ulam stabilities of ordinary differential equations in a Banach space," Carpathian Journal of Mathematics, vol. 26, no. 1, pp. 103-107, 2010.

[49] J. K. Hale and S. M. Verduyn Lunel, Introduction to FunctionalDifferential Equations, Applied Mathematical Sciences series, Springer-Verlag, Berlin, Germany, 1993. 


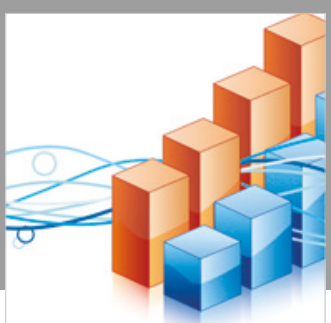

Advances in

Operations Research

\section{-n-m}
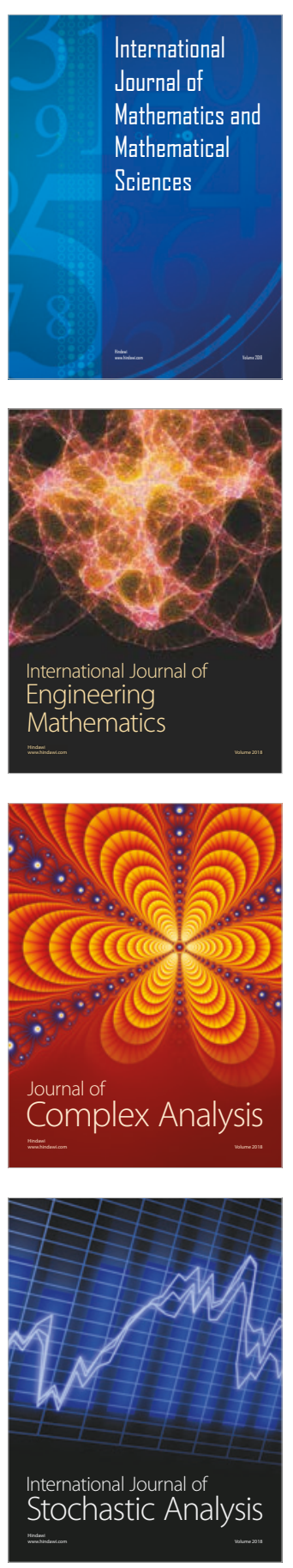
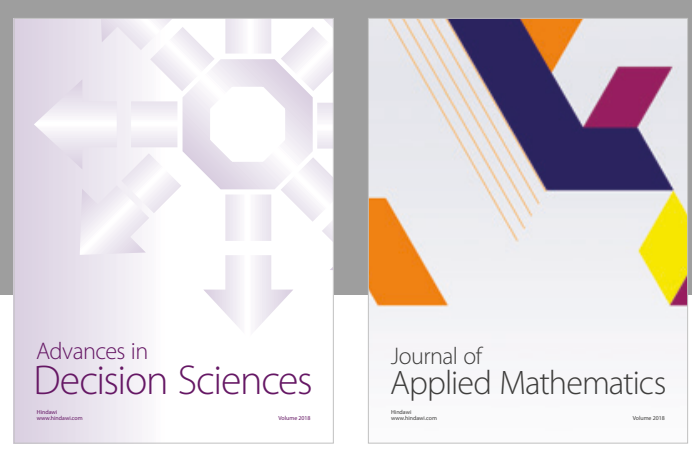

Journal of

Applied Mathematics
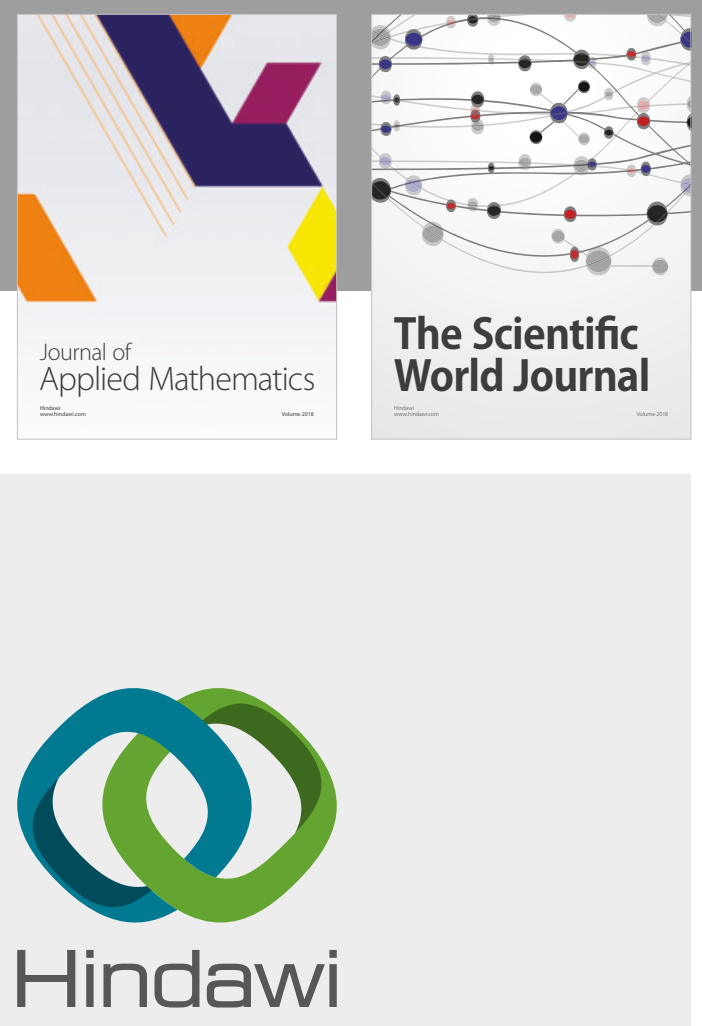

Submit your manuscripts at

www.hindawi.com

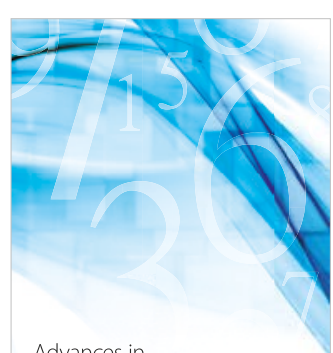

Advances in
Numerical Analysis
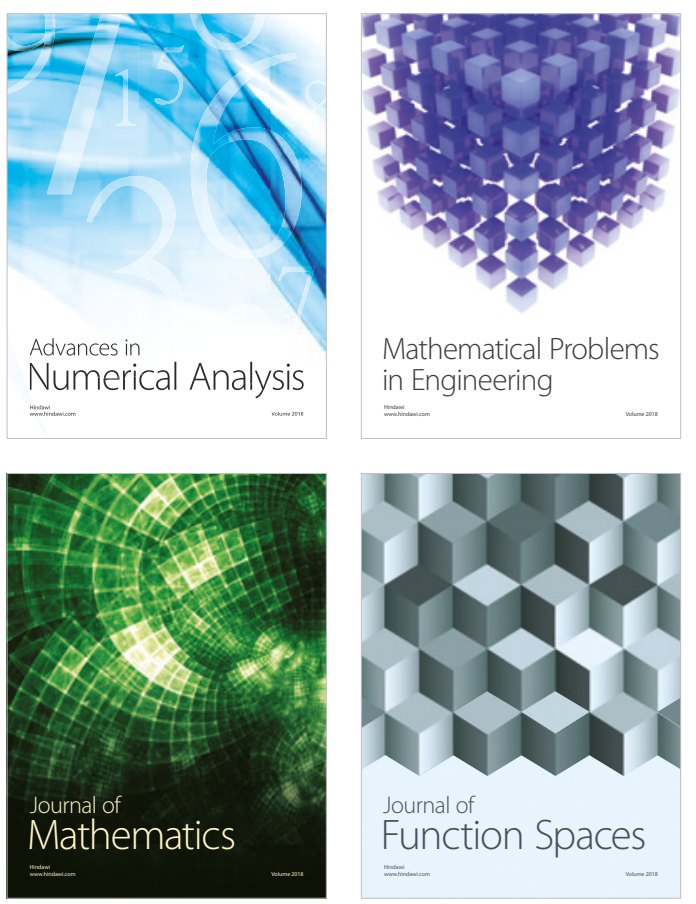

Mathematical Problems in Engineering

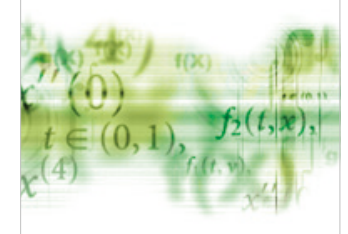

International Journal of

Differential Equations

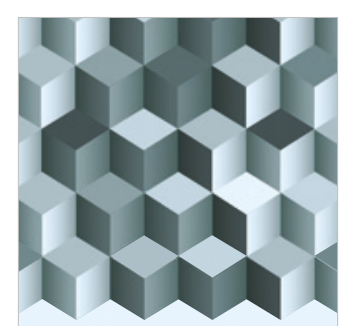

Journal of

Function Spaces
The Scientific

World Journal

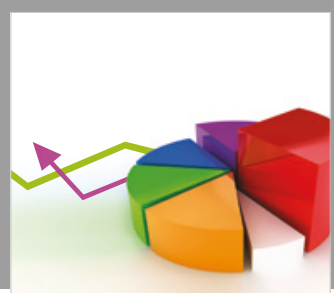

Journal of

Probability and Statistics
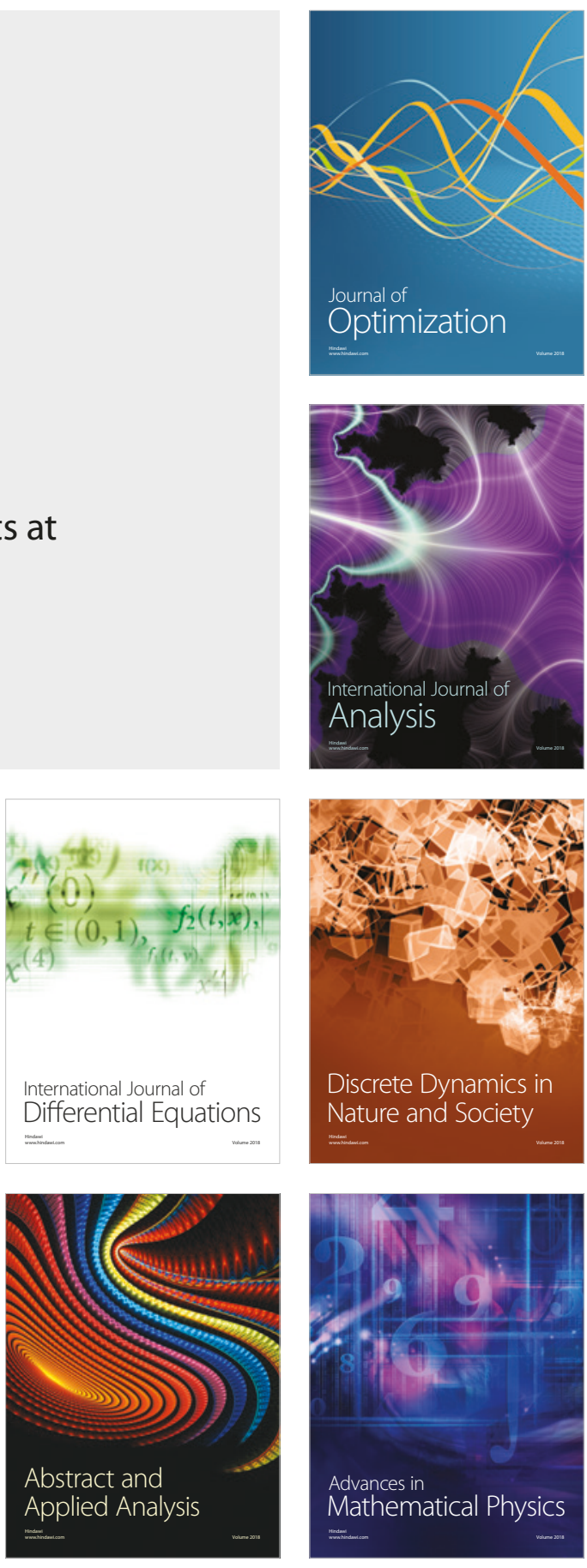